\title{
Impact of lattice dynamics on the phase stability of metamagnetic FeRh: Bulk and thin films
}

\author{
M. Wolloch, ${ }^{1, *}$ M. E. Gruner, ${ }^{2}$ W. Keune, ${ }^{2}$ P. Mohn, ${ }^{1}$ J. Redinger, ${ }^{1}$ F. Hofer, ${ }^{3}$ D. Suess, ${ }^{3}$ R. Podloucky, ${ }^{4}$ J. Landers, ${ }^{2}$ \\ S. Salamon, ${ }^{2}$ F. Scheibel, ${ }^{2}$ D. Spoddig, ${ }^{2}$ R. Witte, ${ }^{5}$ B. Roldan Cuenya, ${ }^{6}$ O. Gutfleisch, ${ }^{7}$ M. Y. Hu, ${ }^{8}$ J. Zhao, ${ }^{8}$ T. Toellner, ${ }^{8}$ \\ E. E. Alp, ${ }^{8}$ M. Siewert, ${ }^{2}$ P. Entel, ${ }^{2}$ R. Pentcheva, ${ }^{2}$ and H. Wende ${ }^{2}$ \\ ${ }^{1}$ Institute of Applied Physics, Vienna University of Technology, Wiedner Hauptstr. 8-10/134, 1040 Vienna, Austria \\ ${ }^{2}$ Faculty of Physics and Center for Nanointegration Duisburg-Essen (CENIDE), University of Duisburg-Essen, 47048 Duisburg, Germany \\ ${ }^{3}$ Institute of Solid State Physics, Vienna University of Technology, Wiedner Hauptstr. 8-10/134, 1040 Vienna, Austria \\ ${ }^{4}$ Institute of Physical Chemistry, University of Vienna, Sensengasse 8/7 A-1090 Vienna, Austria \\ ${ }^{5}$ Institute of Nanotechnology, Karlsruhe Institute of Technology, 76344 Eggenstein-Leopoldshafen, Germany \\ ${ }^{6}$ Department of Physics, Ruhr-University Bochum, 44780 Bochum, Germany \\ ${ }^{7}$ Materials Science, TU Darmstadt, 64287 Darmstadt, Germany \\ ${ }^{8}$ Advanced Photon Source, Argonne National Laboratory, Argonne, Illinois 60439, USA
}

(Received 24 August 2016; published 22 November 2016)

\begin{abstract}
We present phonon dispersions, element-resolved vibrational density of states (VDOS) and corresponding thermodynamic properties obtained by a combination of density functional theory (DFT) and nuclear resonant inelastic x-ray scattering (NRIXS) across the metamagnetic transition of B2 FeRh in the bulk material and thin epitaxial films. We see distinct differences in the VDOS of the antiferromagnetic (AF) and ferromagnetic (FM) phases, which provide a microscopic proof of strong spin-phonon coupling in FeRh. The FM VDOS exhibits a particular sensitivity to the slight tetragonal distortions present in epitaxial films, which is not encountered in the AF phase. This results in a notable change in lattice entropy, which is important for the comparison between thin film and bulk results. Our calculations confirm the recently reported lattice instability in the AF phase. The imaginary frequencies at the $X$ point depend critically on the Fe magnetic moment and atomic volume. Analyzing these nonvibrational modes leads to the discovery of a stable monoclinic ground-state structure, which is robustly predicted from DFT but not verified in our thin film experiments. Specific heat, entropy, and free energy calculated within the quasiharmonic approximation suggest that the new phase is possibly suppressed because of its relatively smaller lattice entropy. In the bulk phase, lattice vibrations contribute with the same sign and in similar magnitude to the isostructural AF-FM phase transition as excitations of the electronic and magnetic subsystems demonstrating that lattice degrees of freedom need to be included in thermodynamic modeling.
\end{abstract}

DOI: 10.1103/PhysRevB.94.174435

\section{INTRODUCTION}

During recent years, ordered B2 $\mathrm{FeRh}$ ( $\mathrm{CsCl}$ structure) has received increased attention due to its extraordinary properties, in particular its temperature-driven isostructural transition between a ferromagnetic (FM) and antiferromagnetic (AF) phase at $T_{\mathrm{M}} \sim 350 \mathrm{~K}$, which was discovered more than seven decades ago [1-4]. This transition is accompanied by a large volume change of $\sim 1 \%$ and a complete loss of the Rh moment in the AF phase $\left(1.0 \mu_{\mathrm{B}}\right.$ in FM), while the Fe moment remains large and essentially constant around 3.2-3.3 $\mu_{\mathrm{B}}$ across the transition. The availability of a metamagnetic transition near room temperature (RT) makes FeRh an interesting material for a number of technological applications like heat assisted magnetic recording (HAMR) [5], antiferromagnetic spintronics [6], and magnetic refrigeration [7]. For a recent review on this topic see Ref. [8].

HAMR is believed to be the future magnetic recording technology in order to extend the areal density to $4 \mathrm{~Tb} / \mathrm{in}^{2}$ and

\footnotetext{
*mwo@cms.tuwien.ac.at
}

Published by the American Physical Society under the terms of the Creative Commons Attribution 3.0 License. Further distribution of this work must maintain attribution to the author(s) and the published article's title, journal citation, and DOI. beyond [5]. Near-field transducers (NFC) are used to focus laser light well below the diffraction limit to give a thermal write assist, enabling the use of highly anisotropic recording media like FePt $[9,10]$. Due to the high Curie temperature of $\mathrm{FePt}$, thermally written-in-errors due to highly excited states in combination with low saturation magnetization, as well as the limited lifetime of NFCs remain an issue for this technology $[11,12]$. An interesting idea to overcome those problems is to replace the second-order transition of FePt by the first-order transition of FeRh. Thiele et al. proposed an exchange spring structure for the recording process by coupling FeRh to FePt [13]. The advantage is that the first-order phase transition of FeRh can be tailored well below the Curie temperature of FePt, which relaxes the lifetime and reliability problem of the NFCs. Furthermore, during recording, the magnetic moment of FeRh is still high overcoming the problem of thermally written-in errors [14].

Another exciting application of FeRh is in the developing field of spintronics, which is promising significant advantages in data storage [15]. It has been shown that epitaxial coupling of FeRh films to ferroelectric substrates allows the $\mathrm{AF}$ to FM transition in FeRh to be driven by electric fields, leading to electric on and off switching of ferromagnetism near RT [16-19]. In contrast to FM memory and storage devices, applications based on AF spintronics are insensitive to magnetic field perturbation and generate no magnetic stray fields, thus eliminating crosstalk. These advantages 
come at the price of an increased difficulty in manipulating the antiferromagnetic aligned magnetic moments [6,20]. A room temperature FeRh AF memory resistor was recently demonstrated by Marti and coworkers, using the FM phase to prealign the magnetic moments of the AF phase after cooling, and using the anisotropic magnetoresistance (AMR) to read out data [21]. Soon after, sequential write-read operations on FeRh AF memory were performed using Joule heating to trigger the metamagnetic transition [22,23]. Employing Mössbauer spectroscopy, Bordel et al. reported a strain-driven Fe-spin reorientation across the AF-to-FM transition in epitaxially strained FeRh thin films grown on $\mathrm{MgO}$, which could be of significant use in AF spintronics applications [24]. It has also been shown that FeRh can be used as a material for spin wave transmission [25].

Recently, also the giant magnetocaloric effect (MCE) of B2 FeRh shifted back into the scientific focus, since FeRh has one of the highest adiabatic temperature changes of all known materials [26-31], which in addition is accompanied by large elasto- and barocaloric effects [32-34]. However, the transition suffers from a large hysteresis and a high sensitivity to stoichiometry and antisite defects [35]. Nonetheless, Liu et al. reported a large reversible caloric effect in a dual-stimulus magnetic-electric refrigeration cycle for a FeRh film grown epitaxially on $\mathrm{BaTiO}_{3}$ [36].

The origin of the metamagnetic transition has been under vibrant debate for more than half a century. An early explanation was given using the exchange inversion model by Kittel, where the exchange parameters vary linearly with the lattice parameter and change their sign at some critical value, thus causing the transition [37]. However, this model is incompatible with the large entropy change observed at the transition [38-41]. Later, reasoned by the large differences in low-temperature specific heats between the AF and FM phases, Tu et al. [42] argued that a change in entropy of band electrons is solely responsible for the transition, but this view fails to explain the transition in the case of admixture of 5\% Ir to FeRh, where the relation of the specific heats becomes reversed $[38,43,44]$.

In 2003, part of the present authors proposed an explanation for the transition based on the competition between AF Fe-Fe and FM Fe-Rh exchange interactions. Monte Carlo simulations of a Blume-Capel spin model suggested that longitudinal thermal fluctuations of the Rh magnetic moments in the FM phase give rise to a Schottky anomaly far below $T_{\mathrm{M}}$, which finally provides the entropic stabilization of the FM phase [45]. Coupling the magnetic subsystem to lattice and volume degrees of freedom leads to a very good agreement with experiment in terms of the entropy change $\Delta S$ at $T_{\mathrm{M}}$ and the temperature dependence of the Gibbs free energy difference $\Delta G$. Recent state-of-the-art measurements of the specific heat $C_{p}$ in FeRh thin films with AF and FM magnetic order seem to support the presence of this Schottky anomaly [46]. However, Cooke et al. separated the magnetic contribution from the other degrees of freedom in terms of a simple Debye model due to the lack of reliable lattice vibration data. Based on their analysis these authors proposed a strong competition between large magnetic and lattice contributions to $\Delta S$ with opposite sign.

In 2004, Mryasov showed that it is also possible to model the transition with a Heisenberg Hamiltonian including bi-quadratic exchange interactions $[47,48]$. In contrast to Ref. [45], the Rh moments were treated as induced by the magnetic moments of the surrounding Fe. Derlet introduced an empirical Landau-Heisenberg model with parameters fitted to existing ab initio calculations. The paper concluded that a quadratic exchange term is needed to produce the transition and that both volume and magnetic fluctuations are equally important [49]. Barker and Chantrell extended Mryasov's model by fully expanding the quadratic spin interactions into four spin exchange terms, which were parameterized from experimental data [50]. Solving the Landau-Lifshitz-Gilbert equation using atomistic spin dynamics yields $T_{\mathrm{M}}$ in good agreement with experiment.

The DFT calculations of Sandratskii and Mavropoulos pointed out that in the AF phase hybridization between Fe and $\mathrm{Rh}$ states causes a local spin polarization of $\mathrm{Rh}$, which averages to zero [51]. Later on, Kudrnovský, Drchal, and Turek argued that the hybridization with surrounding Fe moments is the main reason for the development of magnetic moments on $\mathrm{Rh}$ atoms in the FM phase [52]. The importance of hybridization effects in both AF and FM speaks against a simple Stoner-like picture of an induced $\mathrm{Rh}$ moment and rather for the presence of metastable magnetic states of Rh.

Recent work also aimed at incorporating finite temperature changes to the electronic structure arising from magnetic excitations. Deàk and coworkers evaluated the magnetic and electronic contributions to $\Delta G$ in their relativistic disordered local moment (DLM) approach [53]. They were able to reproduce a transition from $\mathrm{AF}$ to $\mathrm{FM}$, albeit at a rather large temperature and atomic volume. In a similar spirit, Polesya et al. evaluated an extended Heisenberg Hamiltonian which only acts on the Fe atoms, where the FM exchange interaction is scaled according to a response function dependent on the $\mathrm{Rh}$ moments [54]. With magnetic exchange parameters self-consistently obtained from uncompensated DLM calculations, which correspond to the average moment of the configuration, they obtain a very reasonable transition temperature of $\sim 320 \mathrm{~K}$.

A completely different route was taken by $\mathrm{Gu}$ and Antropov, who derived the magnetic contributions to the free energy and $\Delta S$ from the magnon (spin wave) spectra calculated from first principles [55]. In this approach, neither Stoner-type nor spin-flip excitations are considered. From the comparison with experimental data, the authors concluded that the magnetic degrees of freedom provide the dominant contribution to the transition.

The relevance of a specific degree of freedom for the metamagnetic transition is reflected in its temperature dependent contribution to the specific heat $C_{p}$ and finally also to $\Delta S$ and $\Delta G$. A direct comparison of these calculated quantities with experimental data is thus inevitable to evaluate a specific model. This requires the detailed knowledge of all individual contributions to the Gibbs free energy difference between the AF and FM phase $\Delta G(T, p)$, which is usually divided into vibrational, magnetic and electronic degrees of freedom $\left(\Delta G=\Delta G^{\mathrm{vib}}+\Delta G^{\mathrm{mag}}+\Delta G^{\mathrm{el}}\right)$. So far, respective computational data are only provided by Refs. $[45,53,55]$. Experimental information on $C_{p}(T)$ is available from Refs. [46,56], while $\Delta G(T)$ was measured by Ponomarev [57]. 
This work aims at providing for the first time a comprehensive overview of the lattice dynamical contribution to the phase stability in FeRh, from both the computational as well as the experimental point of view. With the notable exception of a pioneering inelastic neutron spectroscopy study of the phonon dispersion along the [111] crystallographic direction in the AF state at room temperature [58], and the recent determination of the element-specific Debye-Waller factor by x-ray spectroscopy [59], no experimental study on lattice vibrations of B2 FeRh has been published to the best of our knowledge. Very recently, a computational study reported unstable lattice dynamics in the B2 AF structure in combination with a strain-induced softening of the shear elastic constant $C^{\prime}$ [60]. However, thermodynamic properties associated with the lattice degrees of freedom are still not available, yet.

After an introduction to methodological details (Sec. II), we present a thorough characterization of electronic structure and phonon dispersion relations (Sec. III). The latter imply an instability of the B2 AF phase towards a new monoclinic or orthorhombic low-energy phase, which depends sensitively on the magnitude of the Fe moment. In Sec. IV, we compare the vibrational density of states (VDOS) with results of ${ }^{57} \mathrm{Fe}$ nuclear resonant inelastic $\mathrm{x}$-ray scattering (NRIXS), which deliver the Fe-projected VDOS of B2-ordered AF and FM $\mathrm{FeRh}$ thin films. From the latter, we derive Fe-projected thermodynamic quantities. Finally in Sec. V, we evaluate from first principles the vibrational and electronical contributions $\Delta G^{\mathrm{vib}}(T)$ and $\Delta G^{\mathrm{el}}(T)$ to the metamagnetic transition and for the proposed new low-temperature phase in the quasiharmonic approximation. The calculated results are found to be in excellent agreement with available experimental data.

\section{METHODOLOGICAL DETAILS}

\section{A. Electronic structure calculations}

Spin-polarized DFT computations have been performed employing the Vienna $a b$ - initio simulation package VASP [61-64] version 5.4.1, using the projector augmented-wave (PAW) method [65,66]. A dense $\Gamma$-centered $k$ mesh of $17 \times 17 \times 17$ points was used to sample the Brillouin zone of our four-atom fcc-like unit cell. Meshes of equal or greater density were used for larger supercells. The plane-wave energy cutoff was chosen to be $450 \mathrm{eV}$, more than $160 \%$ (180\%) of the standard value for the Fe (Rh) PAW potential (set of 2003) which treats the $3 d$ and $4 s(4 p, 5 s$, and $4 d)$ electrons as valence. With this $k$ mesh and energy cutoff total energies are converged to less than $1 \mathrm{meV}$ per formula unit. We employ different functionals to describe the effects of exchange and correlation, to study the functional dependence of our results. If not indicated otherwise, the Perdew-Burke-Ernzerhof version of the generalized gradient correction (GGA) (PBE [67]) has been used. In addition, we used the revised PerdewBurke-Ernzerhof (RPBE [68]), the Perdew-Burke-Ernzerhof revised for solids (PBEsol [69]), and the Perdew-Wang 91 (PW91 [70]) GGAs. Moreover, the van der Waals corrected optB86b [71] and the local-density approximation (LDA [72]) functionals have been employed in parts of this work. To ensure accurate forces during relaxations and phonon calculations, we use an additional superfine fast Fourier transform (FFT) grid for the evaluation of the augmentation charges and a smearing of $\leqslant 0.1 \mathrm{eV}$ according to Methfessel and Paxton [73] (first order). For total energy calculations, the tetrahedron method with Blöchl corrections has been used [74]. In all total-energy GGA calculations, we explicitly account for nonspherical contributions of the gradient corrections inside the PAW spheres. Phonon calculations were carried out in the harmonic approximation using both the small displacement method (usually $0.01 \AA$ ) and density functional perturbation theory (DFPT), using the PHONOPY [75] and PHON [76] codes.

For the calculation of the thermodynamic contributions from the vibrational and electronic degrees of freedom in the quasiharmonic approximation, we employ a similar but slightly different setup. We used valence states of $3 p, 3 d$, and $4 s$ for $\mathrm{Fe}$ and $4 p, 4 d$, and $5 s$ for $\mathrm{Rh}$ with a plane wave cutoff $E_{\text {cut }}=450 \mathrm{eV}$. We used a $3 \times 3 \times 3$ supercell and mostly single displacements of about $0.02 \AA$ to keep the numerical effort tractable. Forces were determined using a MonkhorstPack $k$ grid of $4 \times 4 \times 4$ (except for the orthorhombic Pmm2 structure, where, according to the larger primitive cell and the thoroughly stable phonon dispersion, we reduced the $k$ mesh to $2 \times 2 \times 2$ ) in combination with a finite temperature smearing according to Methfessel and Paxton [73] with a broadening of $\sigma=0.1 \mathrm{eV}$. We used the PBE exchange-correlation functional which is a frequent and well corroborated choice to describe structural properties of ferrous alloys. The influence of the exchange and correlation functional on the thermodynamic properties is discussed in detail in Sec. V A of Ref. [77]. For the calculations of the thermodynamic properties from the vibrational density of states, we could safely neglect the imaginary modes in the $\mathrm{B} 2(\mathrm{AF})$ phase. These occur only at small lattice constants $a_{0} \leqslant 3.02 \AA$ and are present only in a very small fraction of the reciprocal space; even when imaginary modes were omitted, the integrated density of states deviates from unity by less than $0.1 \%$. Consequently, a comparison of the thermodynamic quantities with the results of computationally much more demanding calculations for fully relaxed configurations did not result in a notable difference. The thermodynamic quantities of the electronic subsystems were calculated in a similar fashion from a finely resolved electronic density of states, calculated for different volumes with a Monkhorst-Pack $k$ grid of $20 \times 20 \times 20$ and Brillouin zone integration via the tetrahedron method with Blöchl corrections [74]. The finite temperature modeling of the electronic subsystem simply involved the folding of the density of states with the Fermi distribution function. The impact of finite temperature magnetic spin-flip or spin-wave excitations on the electronic structure, which was incorporated in the approach of Déak et al. [53] and Polesya et al. [54], has not been taken into account here.

\section{B. Samples and experimental procedures}

Two FeRh thin-film samples (labeled FeRh02 and FeRh03, respectively) with different stoichiometries were grown by molecular-beam epitaxy (MBE) via codeposition of ${ }^{57} \mathrm{Fe}$ metal and $\mathrm{Rh}$ in ultrahigh vacuum onto clean $\mathrm{MgO}(001)$ substrates held at $300{ }^{\circ} \mathrm{C}$ during deposition. The preselected deposition rates for ${ }^{57} \mathrm{Fe}$ (enriched to $95 \%$ in the isotope ${ }^{57} \mathrm{Fe}$ ) and $\mathrm{Rh}$ were measured and controlled by several independent 
quartz-crystal oscillators. The FeRh film thickness was about $100 \mathrm{~nm}$. After deposition, the films were in situ annealed at $800{ }^{\circ} \mathrm{C}$ (sample FeRh02) or $700{ }^{\circ} \mathrm{C}$ (sample FeRh03) in order to promote the $\mathrm{B} 2$ order. The $\mathrm{B} 2$ structure and the epitaxial (001) growth were verified by ex situ conventional $\Theta-2 \Theta$ $\mathrm{X}$-ray diffraction. The actual composition of the samples was inferred from energy-dispersive $x$-ray spectroscopy (EDX) and $\mathrm{X}$-ray photoelectron spectroscopy (XPS). The composition was found to be $(51.4 \pm 1.2)$ at.\% Fe for sample FeRh02 (i.e., $\left.\mathrm{Fe}_{51} \mathrm{Rh}_{49}\right)$ and $(48.0 \pm 1.0)$ at.\% Fe for sample FeRh03 (i.e., $\mathrm{Fe}_{48} \mathrm{Rh}_{52}$ ), as compared to the nominal composition (according to the quartz-crystal oscillators) of 51 at.\% Fe and 48 at.\% Fe, respectively. Structural details were studied by high-resolution $\mathrm{x}$-ray diffraction using a four-circle diffractometer. This diffractometer allowed the determination of the out-of-plane (c) and in-plane (a) lattice parameters of the FeRh thin films by measuring asymmetric reflections, thus having an in- and out-of-plane component. We obtained a $c / a$ ratio of 1.0114(7) for sample FeRh02 $\left(\mathrm{Fe}_{51} \mathrm{Rh}_{49}\right)$ and 1.0057(7) for sample FeRh03 $\left(\mathrm{Fe}_{48} \mathrm{Rh}_{52}\right)$, showing that the $c / a$ ratio is slightly larger for sample $\mathrm{Fe}_{51} \mathrm{Rh}_{49}$ than for sample $\mathrm{Fe}_{48} \mathrm{Rh}_{52}$. Our room temperature (RT) $c / a$ values are in good agreement with experimental $c / a$ values of 1.016 for $\mathrm{FM} \mathrm{Fe}_{49} \mathrm{Rh}_{51}$ and 1.008 for $\mathrm{AF} \mathrm{Fe}_{49} \mathrm{Rh}_{51}$ epitaxial thin films on $\mathrm{MgO}(001)$ reported by Bordel et al. [24]. The samples were further characterized by ex situ ${ }^{57} \mathrm{Fe}$ conversion-electron Mössbauer spectroscopy (CEMS) and vibrating sample magnetometry (VSM). These results showed that sample FeRh02 $\left(\mathrm{Fe}_{51} \mathrm{Rh}_{49}\right)$ is ferromagnetic (FM) from RT down to $5 \mathrm{~K}$, while sample FeRh03 $\left(\mathrm{Fe}_{48} \mathrm{Rh}_{52}\right)$ is antiferromagnetic (AF) up to $\sim 360 \mathrm{~K}$, where it starts to transform upon heating to the FM state, the transition being completed at $\sim 404 \mathrm{~K}$. Further details on sample preparation and characterization will be published elsewhere [78]. Shortly ( $<1$ month) after sample preparation, the ${ }^{57}$ Fe NRIXS measurements were performed at $\sim 60, \sim 300$, and $\sim 416 \mathrm{~K}$ at the undulator beamline 3-ID at the Advanced Photon Source, Argonne National Laboratory. ${ }^{57}$ Fe NRIXS is selective to the ${ }^{57}$ Fe resonant isotope only and measures the phonon excitation probability, as described in Refs. [79-82]. This provides the Fe-projected (partial) phonon (or vibrational) density of states (VDOS) rather directly with a minimum of modeling [83]. A high-resolution monochromator [84] was used to produce $\mathrm{x}$-ray with meV energy bandwidth for phonon studies. The monochromatized synchrotron radiation was incident onto the thin-film surface under a grazing angle of a few degrees. The synchrotron beam energy was scanned around the resonant energy of the ${ }^{57} \mathrm{Fe}$ nucleus $(14.413 \mathrm{keV})$ with an energy resolution $\Delta E$ of $1.3 \mathrm{meV}$ and was focused onto the sample surface by a Kirkpatrick-Baez mirror. The average collection time per NRIXS spectrum was about 6-8 h. The evaluation of the NRIXS spectra and the extraction of the VDOS was performed using the PHOENIX software by W. Sturhahn [83].

\section{HARMONIC LATTICE VIBRATIONS AND STRUCTURAL STABILITY}

\section{A. Results}

In the ordered cubic $\mathrm{B} 2$ structure ( $\mathrm{CsCl}$ prototype) $\mathrm{FeRh}$ exhibits two major magnetic configurations, AF coupling (between the $\{111\}$ planes) and FM coupling, which are close

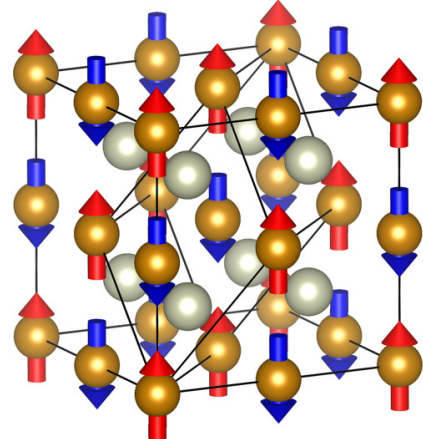

(a)

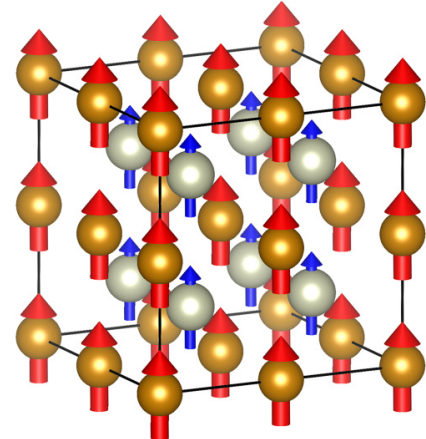

(b)
FIG. 1. Magnetic configurations of FeRh. Iron atoms are shown in gold, rhodium in silver. (a) B2(AF) (also called AFM-II or AFM-G) configuration with the fcc-like unit cell shown. Fe atoms in the $\{111\}$ lattice planes are alternating spin-up (red) and spin-down (blue). (b) $\mathrm{B} 2(\mathrm{FM})$ state where also the $\mathrm{Rh}$ atoms possess a magnetic moment (blue, smaller than the iron moments which are shown in red).

in energy and are visualized in Fig. 1. Initially, calculations where carried out with the PBE GGA functional, and, in accordance with literature, the magnetic ground state for FeRh was found to be the AF configuration, where the Fe atoms are coupled ferromagnetically within the $\{111\}$ planes with alternating alignment of the spins in adjacent planes. In this configuration, the rhodium atoms do not carry a magnetic moment. The FM configuration is slightly higher in energy $(+70.8 \mathrm{meV} /$ f.u. compared to AF) and has a larger equilibrium volume. Here, the rhodium atoms also possess a magnetic moment, which appears to be induced by the Fe atoms. Details can be found in Table I.

Our ground-state lattice constant of $a_{\mathrm{AF}}=2.990 \AA$ at $0 \mathrm{~K}$ is in good agreement with the experimental values (2.986 $\AA$ [4], $2.993 \AA$ [85], and $3.000 \AA$ [86]) and previous calculations (2.996 $\AA$ [55], $2.998 \AA$ [87], and $3.002 \AA[45,60])$. The calculated lattice parameter for the $\mathrm{FM}$ structure at $0 \mathrm{~K}$ is, at $a_{\mathrm{FM}}=3.007 \AA$, also in good agreement with previous work (3.020 ̊ [45], 3.012 ̊ [87], and 3.018 $\AA$ [55,60]).

Earlier investigations reported that the $\mathrm{B} 2(\mathrm{AF})$ phase is soft with respect to a tetragonal distortion corresponding to the martensitic Bain path from the body centered cubic (bcc) to the face centered cubic (fcc) structure $[16,60,88]$. Investigating the Bain path and optimizing the volume of the cell at each step we confirm a second minimum at $c / a=1.247$ (pure fcc: $c / a=\sqrt{2} \simeq 1.414$ ) after overcoming a barrier of

TABLE I. Energy difference to the B2(AF) structure, lattice constants, cell volume, and local magnetic moments of the two studied magnetic configurations of FeRh. Energies and volumes are given per formula unit.

\begin{tabular}{lccccc}
\hline \hline & $\Delta E(\mathrm{meV})$ & $a(\AA)$ & $V\left(\AA^{3}\right)$ & $m_{\mathrm{Fe}}^{\text {loc }}\left(\mu_{\mathrm{B}}\right)$ & $m_{\mathrm{Rh}}^{\text {loc }}\left(\mu_{\mathrm{B}}\right)$ \\
\hline $\mathrm{B} 2(\mathrm{AF})$ & 0 & 2.990 & 26.73 & \pm 3.118 & 0 \\
$\mathrm{~B} 2(\mathrm{FM})$ & 70.8 & 3.007 & 27.20 & 3.177 & 1.058 \\
\hline \hline
\end{tabular}




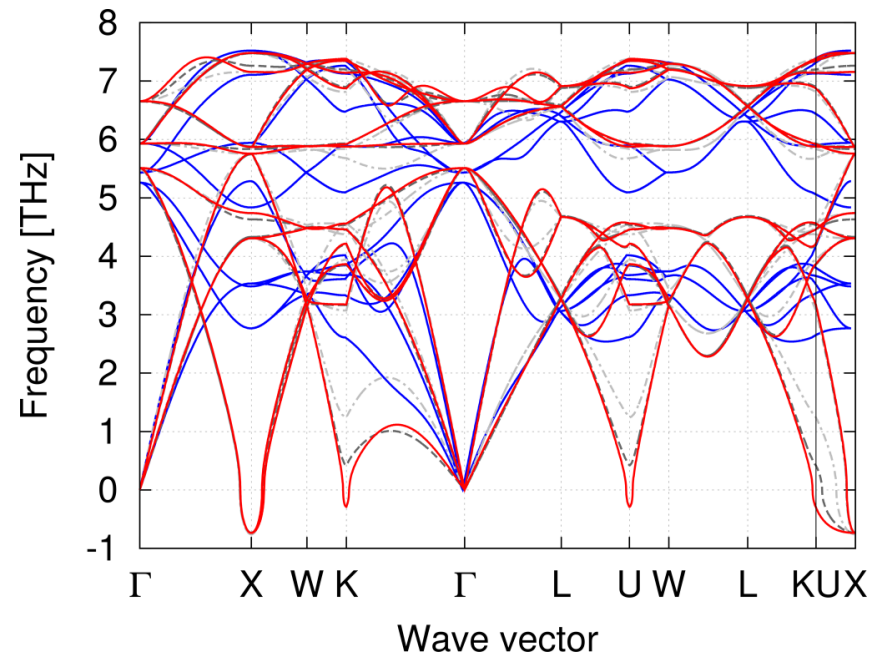

FIG. 2. Phonon band structures for B2 FeRh. Solid red (blue) lines are for $4 \times 4 \times 4$ supercells in the $\mathrm{AF}(\mathrm{FM})$ magnetic configuration. Light grey dashed-dotted lines are results from $2 \times 2 \times 2$, dark grey dashed lines are for $3 \times 3 \times 3 \mathrm{AF}$ supercells. Imaginary frequencies are plotted as negative. The corresponding Brillouin zone can be found in Fig. 1 of Ref. [77].

only $\sim 2 \mathrm{meV} /$ f.u. at $c / a \simeq 1.1$. $^{1}$ Indeed, x-ray diffraction experiments found a transition to a mixture of body centered tetragonal (bct) and fcc under a small uniaxial pressure of $0.25 \mathrm{GPa}$ and by admixture of $\mathrm{Pt}$ or Pd [89-93], and the careful analysis of the elastic behavior in Ref. [60] reveals that the tetragonal shear constant $C^{\prime}$ exhibits a marked softening under compressive volumetric and epitaxial strain. Recently, Kim et al. analyzed tetragonal distortions of various magnetic phases of FeRh in detail and found a new AFM-A' phase, with $c / a=1.26, \sim 7 \mathrm{meV}$ below the cubic B2(AF) phase [94]. A structural transition was also observed in thin films of disordered FeRh by Witte and coworkers [95].

\section{Phonon calculations}

To determine the phononic contribution to the magnetic phase transition described in Sec. I, we calculated the phonon band structure for the two cubic magnetic phases, FM and AF. For better comparison, we used the same primitive cell with fcc basis vectors sketched in Fig. 1(a) for both magnetic phases. Convergence of the stable branches with respect to the supercell size was achieved at $4 \times 4 \times 4$ multiplication of the 4 atom unit cell, which then contains 256 atoms (see Fig. 2).

While the B2(FM) dispersion is stable in the entire Brillouin zone, a region around the $X$ point of the B2(AF) phonon band structure shows imaginary (plotted as negative) frequencies for all cell sizes (see Fig. 2). This indicates a dynamic instability of the crystal and suggests that displacing

\footnotetext{
${ }^{1}$ In contrast to Ref. [60], we find this distorted structure to be slightly lower in energy by $\sim 5 \mathrm{meV} /$ f.u. than the bcc phase using the PBE functional. This result did not change after increasing the plane-wave cutoff to $550 \mathrm{eV}$ as used in Ref. [60]. For the pure fcc phase the energy is higher than bcc by $74.4 \mathrm{meV} /$ f.u. at zero pressure.
}

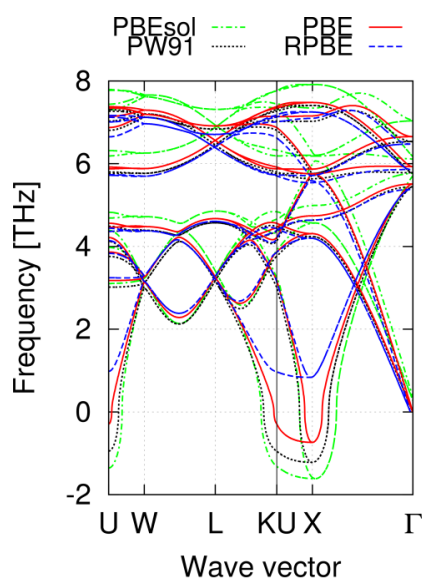

(a)

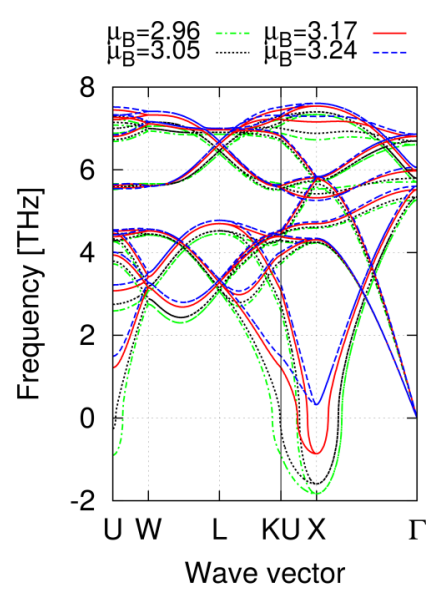

(b)
FIG. 3. (a) Phonon band structures for B2(AF) FeRh calculated for different GGAs. All GGAs other than RPBE show dynamical instabilities. (b) Qualitative PBE phonon band structures for B2(AF) FeRh calculated for constrained magnetic moments. Increasing the magnetic moments on the $\mathrm{Fe}$ atoms from $\pm 2.96 \mu_{\mathrm{B}}$ (green dashdotted line) to $\pm 3.24 \mu_{\mathrm{B}}$ (blue dashed line) gradually stabilizes the whole band structure. If dotted, dash-dotted or dashed lines appear solid, it is due to degenerate bands.

the ions according to the wave vector at $X$ would not result in a restoring force but lead to a lowering of the total energy. The wave vector at $X$, which points along the direction of one of the cubic axes, describes a (doubly degenerate) transverse optical phonon with a periodicity of $2 a_{\mathrm{AF}}$. Comparing results for different supercell sizes shows that the instability does not depend on the cell size along the $\Gamma-X-W$ direction of the Brillouin zone, but spreads out along $X-U$ and $X-K$ with increasing cell size until also $U$ and $X$ are included in the imaginary pocket for the $4 \times 4 \times 4$ supercell.

Phonon calculations require very accurate forces and thus we repeated our volume optimization and phonon calculations with significantly higher plane wave cutoff $(850 \mathrm{eV})$ and $k$-mesh density (corresponding to a $24 \times 24 \times 24$ mesh for the unit cell). The phonon band structure obtained with these parameters showed no significant deviation from Fig. 2, ruling out insufficiently converged computational parameters as a source of the imaginary frequencies.

To investigate whether the instability depends on the applied functional (PBE), we performed additional calculations with other GGAs, first, computing the equilibrium volume and then determining the phonon band structure for PBEsol, PW91, and RPBE. The relevant parts of these phonon band structures are plotted in Fig. 3(a) and significant differences are revealed for the four tested GGA functionals.

Although the stable phonon branches (both acoustic and optical) are in good qualitative agreement, the phonon instability previously detected for PBE is not a universal feature. For PBEsol and PW91, the imaginary pocket around the $X$ point is significantly broader and deeper. It clearly encompasses also the $U$ and $K$ points, where the PBE bands remain nearly stable. On the other hand, the phonon band structure calculated with the RPBE functional is stable at all wave vectors although it also shows considerable softening of the transverse optical modes at the $X, K$, and $U$ points. To further 
TABLE II. Comparison of lattice parameter $a$, cell volume $V$, local magnetic iron moments $m_{\mathrm{Fe}}^{\text {loc }}$, and phonon stability for the calculation of FeRh in the cubic AF phase with different functionals. $\mathrm{PBE}^{*}$ is a $\mathrm{PBE}$ phonon calculation at higher (nonequilibrium) volume.

\begin{tabular}{lcccc}
\hline \hline & $a(\AA)$ & $V\left(\AA^{3} /\right.$ f.u. $)$ & $m_{\mathrm{Fe}}^{\text {loc }}\left(\mu_{\mathrm{B}}\right)$ & Instabilities at \\
\hline LDA & 2.915 & 24.77 & \pm 2.835 & $X, U, K$ \\
optB86b & 2.965 & 26.07 & \pm 3.036 & $X, U, K$ \\
PBEsol & 2.947 & 25.60 & \pm 3.040 & $X, U, K$ \\
PW91 & 2.992 & 26.78 & \pm 3.095 & $X, U, K$ \\
PBE & 2.990 & 26.73 & \pm 3.117 & $X, U, K$ \\
PBE* & 3.014 & 27.39 & \pm 3.152 & $X$ \\
RPBE & 3.014 & 27.39 & \pm 3.183 & \\
Exp. [4] & 2.986 & 26.63 & \pm 3.3 & - \\
Exp. [85] & 2.993 & 26.81 & - & - \\
Exp. [86] & 3.000 & 27.00 & - & - \\
\hline \hline
\end{tabular}

analyze this unsatisfactory result we performed additional volume optimization and phonon calculations for the local density approximation (LDA) and the van der Waals corrected optB86b functionals, as well as a PBE phonon calculation at the higher volume predicted by the RPBE functional. Both the LDA and the optB86b calculations lead to a smaller volume and smaller magnetic moments than all GGAs and both lead to large imaginary pockets around $X$ encompassing also $U$ and $K$, while the PBE calculation at the higher volume (PBE* in Table II) leads to an increased magnetic moment and has only a very small pocket at the $X$ point in the phonon band structure. The results of all calculations are given in Table II, sorted from top to bottom according to increasing magnetic moments. We see [also note Fig. 3(a)] that an increase in magnetic moment is stabilizing the $\mathrm{B} 2(\mathrm{AF})$ phase, as the imaginary pocket is reduced in size from PBEsol $\left( \pm 3.04 \mu_{\mathrm{B}}\right)$ and PW91 $\left( \pm 3.10 \mu_{\mathrm{B}}\right)$, over PBE $\left( \pm 3.12 \mu_{\mathrm{B}}\right)$, to $\mathrm{PBE}^{*}\left( \pm 3.15 \mu_{\mathrm{B}}\right)$, and finally vanishes for RPBE $\left( \pm 3.18 \mu_{\mathrm{B}}\right)$.

Comparing the GGA results, the increased magnetic moment is correlated with an increased atomic volume. For instance, RPBE, at $27.4 \AA^{3} /$ f.u., predicts a significantly higher volume than PBE (26.7 $\AA^{3} /$ f.u.) and PW91 (26.8 $\AA^{3} /$ f.u.). In turn, a calculation with $\mathrm{PBE}$ at an artificially higher volume (labeled PBE* in Table II) also led to a nearly stable phonon band structure. To separate the influence of the magnetic moment and volume on the imaginary mode, we performed PBE phonon calculations with constrained magnetic moments using $2 \times 2 \times 2$ supercells at the equilibrium PBE volume of $26.7 \AA^{3}$. Although we were not able to converge the constrained calculations to the same accuracy as our other phonon calculations, Fig. 3(b) indicates that the increase of the magnetic moments alone is sufficient to stabilize the cubic structure. This observation is in accordance with the results by Aschauer et al. [60], who find that the second minimum along the martensitic Bain path disappears if the local Fe moments are increased. In contrast to our work, however, they use a $\mathrm{PBE}+U$ approach to increase electron localization in the $\mathrm{Fe}$ $d$ orbitals and thus influence the magnetic moments, while we subtly change the hybridization of the orbitals by increasing the size of the magnetic moments directly.

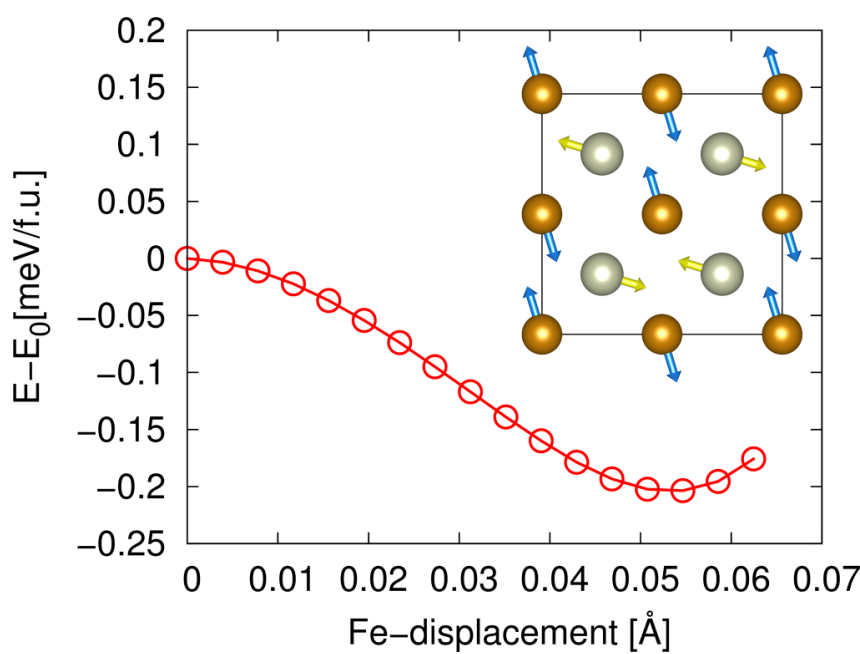

FIG. 4. Frozen phonon calculation corresponding to the unstable phonon at wave vector $X$. Energy is referenced to zero displacements. The displacement is given only for the Fe atoms since it is not equivalent for $\mathrm{Fe}$ and $\mathrm{Rh}$. The inset shows the cubic unit cell and direction of displacements of ions according to the dynamic instability at the $X$ point. Arrows indicate the direction of the displacement, $\mathrm{Fe}$ is shown in gold, $\mathrm{Rh}$ in silver.

While it is interesting that quite small differences in the magnetic moments can lead to a stabilization of the cubic phase, it is certainly true that the size of the magnetic moments and the volume are interrelated effects and an increase of one of them leads to an increase in the other unless some degree of freedom is constrained. Indeed, if the PBE volume is increased to $27.39 \mathrm{~A}^{3}$ the magnetic moments converge to $\pm 3.15 \mu_{\mathrm{B}}$ ( $\mathrm{PBE}^{*}$ in Table II) and the unstable pocket at $X$ is considerably smaller than the one observed if the volume is not increased and only the magnetic moment is constrained to $\pm 3.17 \mu_{\mathrm{B}}$ [see Fig. 3(b)].

\section{Analyzing the phonon instability}

We already mentioned that the instability at the $X$ point in the PBE phonon band structure (see Fig. 2) is a doubly degenerate transverse optical phonon branch with the wave vector pointing along one of the cubic axes. Without loss of generality we chose the $c$ axis as direction of the wave vector. The direction of the ionic displacements resulting from this wave vector is shown in the inset of Fig. 4. The angle of the ion displacements with the cubic axes of the cell is $\sim 17^{\circ}$ and the $\mathrm{Rh}$ atoms become displaced about $16 \%$ less than the $\mathrm{Fe}$ atoms. By stepwise displacing the atoms without allowing cell or atomic relaxations, we see a slight lowering of the total energy and a minimum at an amplitude of about $0.05 \AA$ (see Fig. 4).

While the total reduction in energy is only $\sim 0.2 \mathrm{meV}$ per formula unit, this minimum confirms the phonon calculations and can be used as a starting point for cell- and subsequent ionic relaxations. After carefully relaxing the whole system, we arrive at a monoclinic structure $(P 2 / m)$ with a total energy gain of $24.3 \mathrm{meV}$ per formular unit compared to 


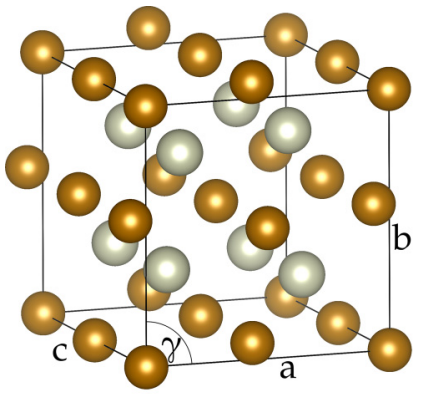

(a)

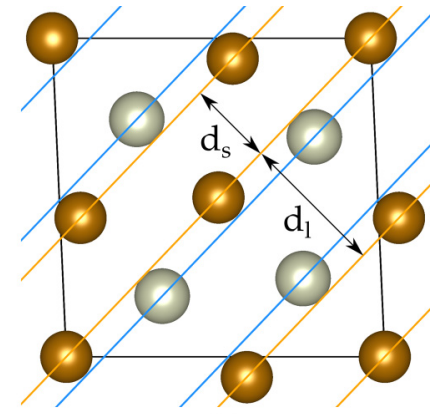

(b)
FIG. 5. (a) Monoclincic unit cell of $P 2 / m$ (AF) FeRh. (b) Side view of the same cell with $\{1 \overline{1} 0\}$ planes drawn for both $\mathrm{Fe}$ (gold) and $\mathrm{Rh}$ (silver) atoms. Arrows indicate short $\left(d_{\mathrm{s}}\right)$ and long $\left(d_{1}\right)$ distance between lattice planes.

B2(AF) (see Fig. 5). ${ }^{2}$ This is more than twice the energy gain reported for a tetragonally distorted structure with similar atomic displacements in Ref. [60].

The change in the angle $\gamma$ to $92.6^{\circ}$ is not particularly large, but the tetragonal distortion is severe, with $c$ compressed by $7.2 \%$ to $5.55 \AA$ and $a$ and $b$ enlarged by $2.8 \%$ to $6.15 \AA$. The distortion of the lattice vectors leads to a reduction in volume by $\sim 2 \%$. The magnetic moments on the $\mathrm{Fe}$ sites are also reduced by $\sim 9 \%$ to $\pm 2.816 \mu_{\mathrm{B}}$ for the $P 2 / \mathrm{m}(\mathrm{AF})$ phase compared to the $\mathrm{B} 2(\mathrm{AF})$ structure. The Rh atoms still do not carry a local moment, a result that was carefully checked by turning all symmetry operations off. In Fig. 5(b), the $\{1 \overline{10}\}$ lattice planes are drawn to clarify in which way the ions are shifted compared to the cubic structure. If one compares Fig. 5(b) with the cubic structure (inset of Fig. 4), it becomes clear that the $\{1 \overline{1} 0\}$ lattice planes, which contain both $\mathrm{Fe}$ and $\mathrm{Rh}$ atoms in the cubic phase, consist of only a single atomic species in the monoclinic phase. Now two closer spaced planes of Fe atoms are followed by two closer spaced planes of Rh atoms, and so forth. The distances between Fe planes (short: $d_{\mathrm{s}}=1.65 \AA$; long: $d_{1}=2.79 \AA$; for Rh planes the situation is exactly the same), indicated by arrows in Fig. 5(b), show the deviation from the cubic structure, where those lattice planes are equidistant with $d_{0}=2.11 \AA$. The stable phonon band structure of the monoclinic phase, as well as crystallographic data can be found in Ref. [77].

Considering the density of states (DOS) for both crystal structures (see Fig. 6), we observe a significant increase of the AF DOS at the Fermi level from 0.6 states/eV/f.u. for the B2 case to 1.4 states/eV/f.u. for the $P 2 / m$ phase, although it is still smaller than the DOS at the Fermi level in the B2(FM) case (1.8 states/eV/f.u.). The loss of symmetry is also clearly visible in the monoclinic DOS, which loses most of the distinct features displayed in the cubic phase and is essentially uniform in the valence band.

\footnotetext{
${ }^{2}$ It is to be noted that the energy landscape of FeRh is rather flat with respect to these atomic displacements and lattice distortions, thus finding the minimum for the monoclinc phase proved to be a rather difficult endeavor, requiring a lot of different relaxation steps and combination of algorithms.
}

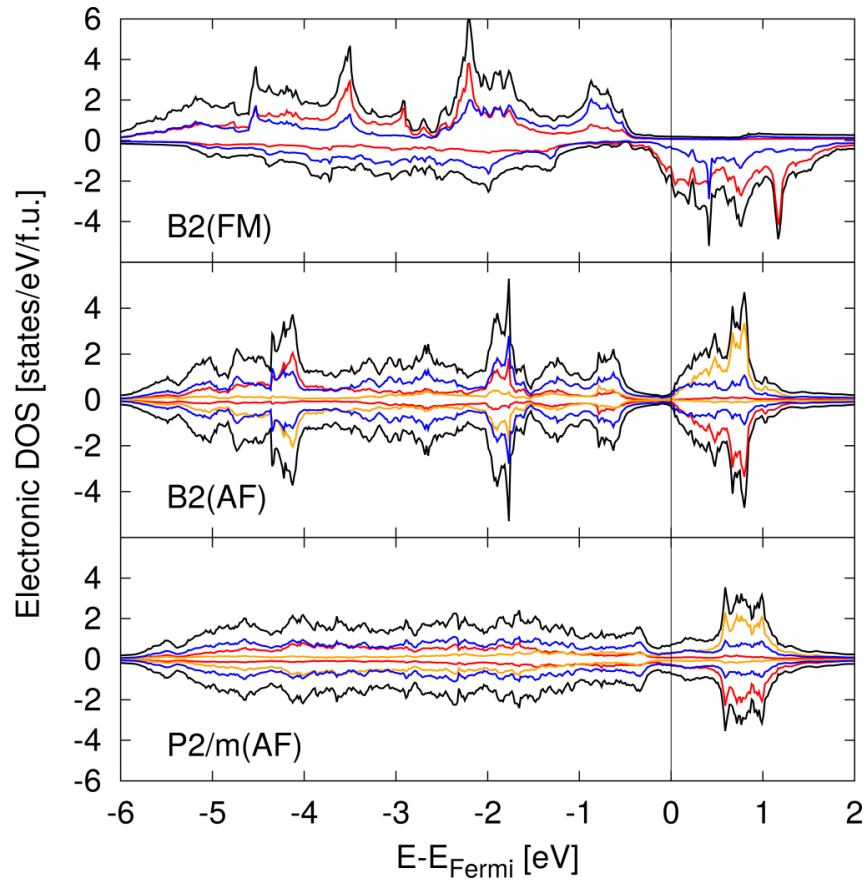

FIG. 6. Electronic DOS for the B2(FM), B2(AF), and $P 2 / m(\mathrm{AF})$ phases of FeRh. Total DOS is plotted in black, Fe states are red and orange (depending on the nonequivalent Fe sites in the AF structure) and $\mathrm{Rh}$ states are blue.

We also investigated the stability of the monoclinic phase with respect to other functionals. To this end, we optimized the volume of the monoclinic cell for all functionals in Table II which show the dynamic instability, while fixing the cell shape and holding the ion positions at their PBE relaxed coordinates. The monoclinic phase is significantly favored in energy for all the functionals, with LDA showing the strongest decrease in energy and PBE the weakest (see Table III). In all cases, the volume and the magnetic moments are significantly reduced, with an average reduction of the volume by $2.2 \%$ and of the local iron moments by $10.9 \%$ for the 3 GGAs PBEsol, PBE, and PW91.

For RPBE, where the B2(AF) structure is stabilized because of the higher magnetic moments and the larger equilibrium volume, the $P 2 / m(\mathrm{AF})$ phase is higher in energy by $4.9 \mathrm{meV} / \mathrm{f}$.u. if only the volume is optimized according to the RPBE functional. If we also allow the ions and the cell shape to

TABLE III. Energy gain $\Delta E$, volume change $\Delta V$, and $\mathrm{Fe}$ local magnetic moment change $\Delta m_{\mathrm{Fe}}^{\text {loc }}$ of the $P 2 / m(\mathrm{AF})$ structure compared to the $\mathrm{B} 2(\mathrm{AF})$ phase for different functionals. Cell shape and ion positions have been relaxed with PBE, but volumes are optimized for each functional.

\begin{tabular}{lccc}
\hline \hline & $\Delta E(\mathrm{meV} /$ f.u. $)$ & $\Delta V(\%)$ & $\Delta m_{\mathrm{Fe}}^{\text {loc }}(\%)$ \\
\hline LDA & -193.1 & -3.4 & -22.8 \\
PBEsol & -87.6 & -2.5 & -13.28 \\
optB86b & -82.3 & -5.0 & -13.6 \\
PW91 & -42.0 & -2.1 & -10.1 \\
PBE & -24.3 & -1.9 & -9.2 \\
\hline \hline
\end{tabular}


relax again with RPBE, we find that the monoclinic phase is almost equivalent in energy (favored by $-0.7 \mathrm{meV} /$ f.u.) to the $\mathrm{B} 2(\mathrm{AF})$ phase for this functional.

The comparison of the different exchange-correlation functionals proves that from the computational point-of-view, the preference for the $P 2 / m(\mathrm{AF})$ ground state is a robust result. Indeed, a closely related relaxation pattern as shown in Fig. 4 (missing the monoclinic distortion) has been found experimentally in ternary bet $\mathrm{FeRh}_{0.38} \mathrm{Pd}_{0.62}$ [96]. Experiments for binary B2 FeRh applying hydrostatic pressures of up to $7 \mathrm{GPa}$ did not reveal any indications for a new phase at room temperature and above in pure FeRh [97,98]. Later experimental work [99] at higher pressures (10 to $20 \mathrm{GPa}$ ) suggests a transition to a fct tetragonal structure with significantly reduced volume, which coexists with the $\mathrm{B} 2$ phase.

The fact that the monoclinic phase has not been found in experiments for pure FeRh until today, although it should be clearly distinguishable from the B2 phase given the large tetragonal distortion $(c / a=0.9)$, requires some discussion. An early measurement by neutron diffraction reports a $\mathrm{Fe}$ moment of $3.30 \mu_{\mathrm{B}}$ [4], which is clearly underestimated by all GGA-type exchange-correlation functionals. According to our constrained moment calculations [see Fig. 3(b)], enhancing the Fe moment to $3.30 \mu_{\mathrm{B}}$ leads to a stable B2(AF) dispersion with standard GGA, even if the lattice constant is fixed at $a_{\mathrm{AF}}=$ $2.990 \AA$ corresponding to the experimental value $[4,85,86]$. However, the GGA is usually known to overestimate magnetic moments [100-104] (rather than to underestimate them) and increased magnetic moments would also destabilize the second fcc like minimum along the martensitic Bain path, which has been experimentally observed for FeRh under high velocity impact deformation or filing $[60,89,105]$. Alternatively, the extremely shallow minimum associated with the unstable phonon (only $0.2 \mathrm{meV} /$ f.u. energy gain in the cubic phase) may be simply smeared out by kinetic fluctuations at temperatures larger than $2 \mathrm{~K}$. We also know from first-principle calculations that a small number of antisite defects are enough to suppress AF order down to low temperatures [35]. It is thus possible that defects and slightly off-stoichiometric compositions also suppress the monoclinic AF phase. Nevertheless, careful adaption of the $c / a$ ratio or application of strain at low temperatures could still lead to a stabilization of the $P 2 / \mathrm{m}$ (AF) low-energy phase with its significantly reduced volume and magnetic moments.

\section{B. Electronic origin of the lattice instability}

Taking into account the magnetic configuration, the FeRh lattice in the cubic AF state belongs to the same point group as the $\mathrm{L} 2{ }_{1}$ Heusler alloys like $\mathrm{Ni}_{2} \mathrm{MnGa}$. These compounds similarly exhibit a soft acoustic phonon in [110] direction, albeit not at the zone boundary point $K$ or even $X$ [108]. The origin of the softening in Ni-Mn-based Heusler compounds is a $\mathrm{Ni}_{u}$ peak right below the Fermi level, which gives rise to extended plane nesting sheets in the Fermi surface connected by the same wave vector describing the soft phonon. As pointed out recently, disordered equiatomic bcc-FeRh under epitaxial strain undergoes a transition to an orthorhombic structure which shows signatures of a martensitic transformation, driven by a redistribution of electronic states away from the Fermi

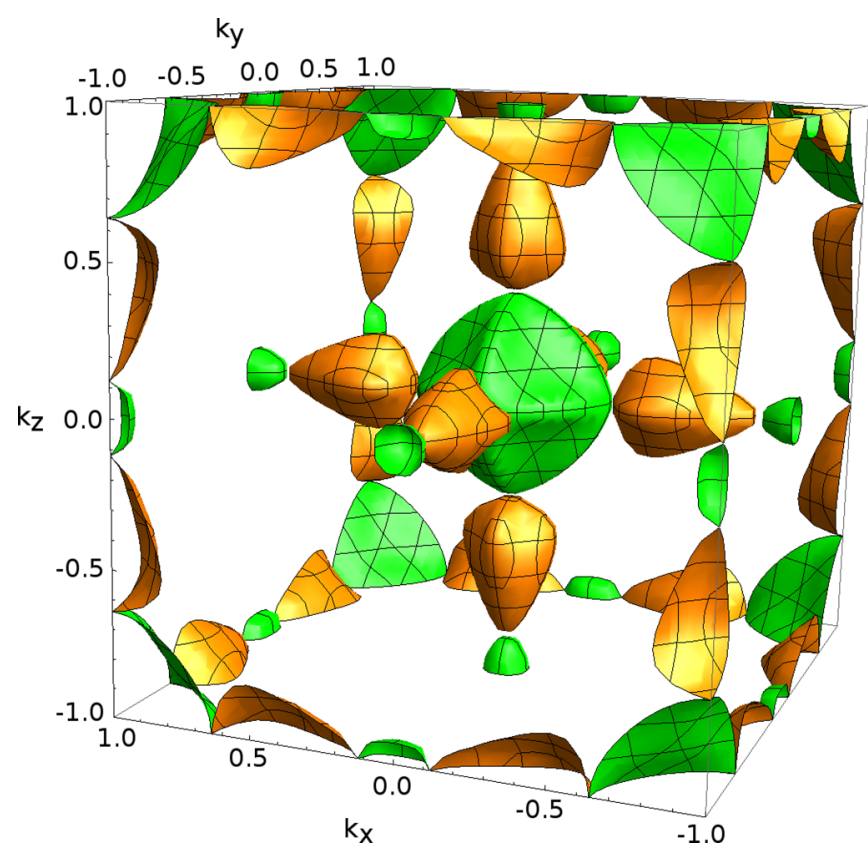

FIG. 7. Fermi surface of B2-ordered AF FeRh in the extended Brillouin zone scheme. The different colors refer to different bands crossing the Fermi level. Clear nesting features are absent. The shape of the Fermi surface agrees well with previous reports. [106,107].

level in combination with the removal of parallel features visible in selected Fermi surface cross-sections [95]. In B2 FeRh, however, the Fermi surface of the AF phase, depicted in Fig. 7 is rather small, which reflects the low density of states at the Fermi level (cf. Fig. 6). It also does not exhibit obvious nesting features. Lowering the energy of the cubic structure according to the soft phonon mode following the path displayed in Fig. 4 does not lead to notable changes in the DOS at the Fermi level. Instead, peaks at 1.9 and $4.2 \mathrm{eV}$ below $E_{\mathrm{F}}$, which arise from flat hybridized d-bands degenerate at $X, L$ and $\Gamma$, split up asymetrically according to the reduced symmetry (see Fig. 4 in Ref. [77]). This reminds of a band-Jahn-Teller mechanism which is taking effect far below the Fermi level. The overall gain in energy is very small and it is impossible to clearly separate the competing contributions. But we may speculate that the strong hybridization of rhodium and iron states in both the AF and the FM phase, responsible for both the implicit splitting of $\mathrm{Rh}$ in the AF phase and the evolution of a net magnetic moment in the FM phase $[51,52]$, is also decisive for the structural stability of B2 FeRh. This could explain why rather different mechanisms such as increasing the local exchange splitting of $\mathrm{Fe}$ via constrained magnetic moments, or decreasing the band width by increasing the volume, or shifting the relative position of the elemental orbitals using the GGA $+U$ approach, have the same consequence, i. e., stabilizing the B2(AF) phase.

An instable phonon can open a downhill relaxation path to a new ground-state structure, as the monoclinic structure for ordered FeRh or the tetragonal $\mathrm{L}_{0}$ phase in the case of the Heusler systems [109,110]. In FeRh, the phonon instability is fragile and is connected to a much smaller energy gain compared to the Heusler alloys. Thus, in addition to the mechanisms discussed above, the relaxation path to the 
monoclinic ground state might easily be blocked by other perturbations as well, like a slight amount of chemical or magnetic disorder, or the presence of lattice defects. However, the phonon-induced modulations are no prerequisite for the existence of a (meta-)stable monoclinic minimum. This is directly seen from a comparison of the electronic density of states (see Fig. 6), which exhibits entirely unrelated features for the B2 and $P 2 / m$ phases, as well as the existence of the minimum for the monoclinic phase for the RPBE functional which predicts stable phonons in $\mathrm{B} 2(\mathrm{AF})$ structure. This suggests that the new phase might be stabilized at low temperatures under carefully designed external conditions, such as a sufficiently large pressure, by epitaxial strain and/or band filling, which tunes the AF-FM transition as well [111].

\section{NUCLEAR RESONANT INELASTIC X-RAY SCATTERING IN THIN FILMS}

Our most important experimental result is presented in Fig. 8. It displays the Fe-projected (partial) VDOS, $g(E)$, of sample $\mathrm{Fe}_{48} \mathrm{Rh}_{52}$ (code FeRh03) measured at $T=59,305$, and $416 \mathrm{~K}$, i.e., across the AF to FM phase transition. The NRIXS raw data and the excitation probabilities (after subtraction of the elastic peak) of this sample, and also of our FM sample $\mathrm{Fe}_{51} \mathrm{Rh}_{49}$ (code FeRh02), are shown in Figs. 5 and 6 of Ref. [77]. In Fig. 8(a), the VDOS of $\mathrm{Fe}_{48} \mathrm{Rh}_{52}$ at $59 \mathrm{~K}$ (when the sample is in the AF state) is characterized by three prominent phonon peaks: a pronounced sharp high-energy peak $\left(\mathrm{P}_{1}\right)$ at $(31.0 \pm 0.2) \mathrm{meV}$, a less pronounced medium-energy peak $\left(\mathrm{P}_{2}\right)$ at $(25.5 \pm 0.3) \mathrm{meV}$, and a very broad, weak low-energy peak $\left(\mathrm{P}_{3}\right)$ at $(16.3 \pm 0.5) \mathrm{meV}$. Upon heating from 59 to $305 \mathrm{~K}$, where the sample is still in the AF state, the overall shape of the VDOS essentially remains the same [Fig. 8(b)], however, we observe a slight red shift (about $1 \%$, averaged over the spectrum) due to the effect of lattice thermal expansion. The redshift can be more clearly seen in Fig. 7(b) of Ref. [77], where the experimental VDOS of $\mathrm{AFFe}_{48} \mathrm{Rh}_{52}$ at 59 and $305 \mathrm{~K}$ are directly compared. When the $\mathrm{Fe}_{48} \mathrm{Rh}_{52}$ sample is heated to $416 \mathrm{~K}$, the VDOS [Fig. 8(c)] distinctly changes in two aspects: (i) the height of the high-energy VDOS peak $\mathrm{P}_{1}$ is drastically reduced to the height of $\mathrm{P}_{2}$ while its width increases, and (ii) the broad (but weak) low-energy feature $\mathrm{P}_{3}$, centered at $\sim 16 \mathrm{meV}$, becomes remarkably narrower. In fact, the largest relative change in the broad feature $\mathrm{P}_{3}$ upon heating to $416 \mathrm{~K}$ occurs at $\sim 10 \mathrm{meV}$, implying a reduction of an apparent shoulder $\left(\mathrm{P}_{4}\right)$ at $(10.0 \pm 1.5) \mathrm{meV}$ that exists at 305 and $59 \mathrm{~K}$, but not at $416 \mathrm{~K}$. Since $T=416 \mathrm{~K}$ is above the transition temperature of $\sim 380 \mathrm{~K}$, the sample $\mathrm{Fe}_{48} \mathrm{Rh}_{52}$ is in the FM state. This transition is clearly seen in the differences between the VDOS in Figs. 8(a)-8(c). Apparently, the phonon spectrum of B2ordered FeRh depends on the type of magnetic ordering (AF or FM). Since the transition occurs isostructurally, the drastic magnetism-dependent modification of the VDOS observed in Fig. 8 is an atomistic manifestation of strong magnetoelastic (or spin-phonon) interaction in the magnetocaloric FeRh compound. In this respect, the FeRh alloy behaves similarly to the magnetocaloric ordered $\mathrm{La}(\mathrm{Fe}, \mathrm{Si})_{13}$ compound, for which also a distinct magnetic-order-dependent modification of the Fe-projected VDOS has been discovered by NRIXS [112].

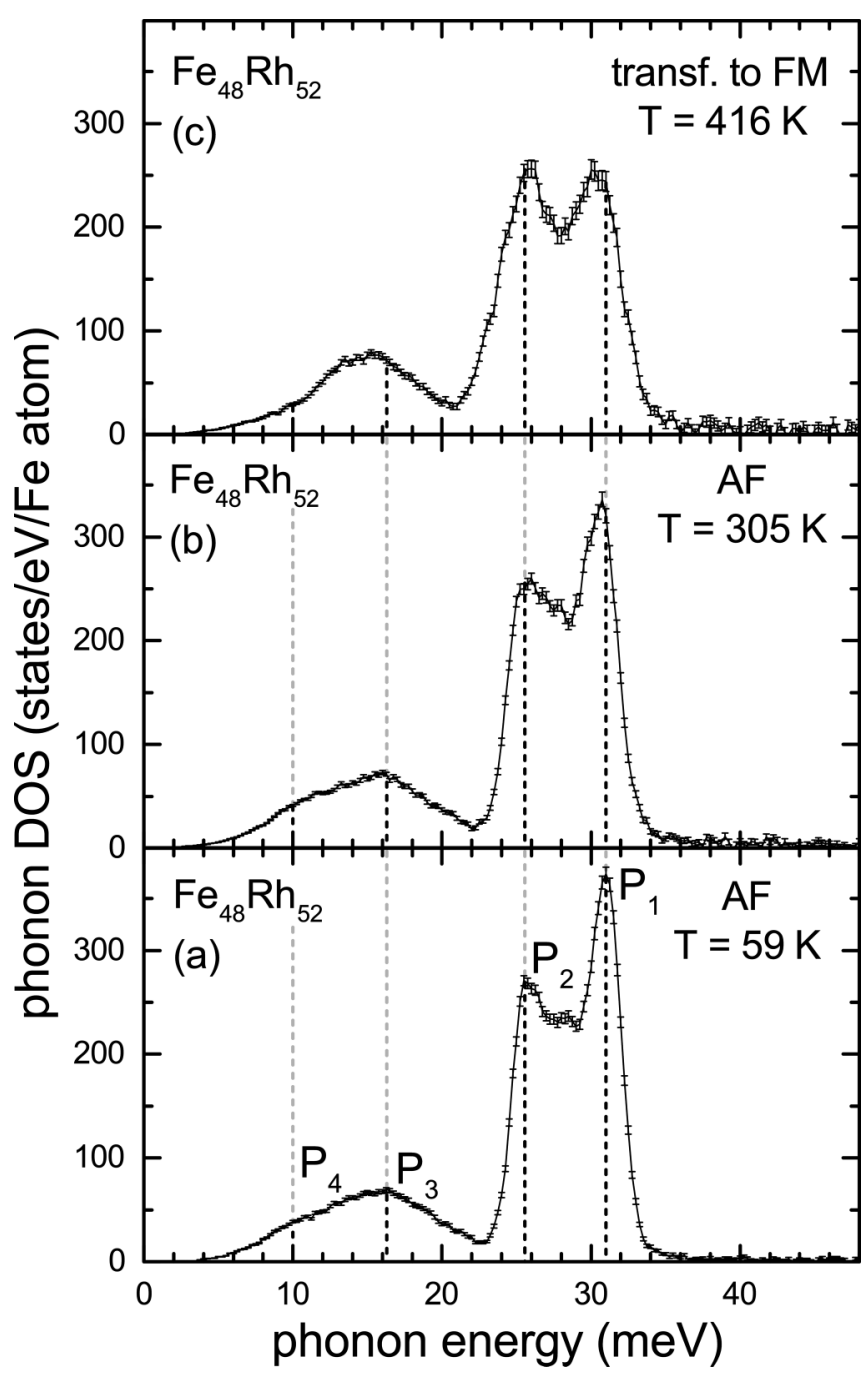

FIG. 8. Fe-projected (partial) VDOS of $\mathrm{Fe}_{48} \mathrm{Rh}_{52}$ (sample code FeRh03) measured by ${ }^{57} \mathrm{Fe}$ NRIXS at (a) $T=59$ (AF), (b) 305 (AF), and (c) $416 \mathrm{~K}(\mathrm{FM})$. The approximate positions of peaks $\mathrm{P}_{1}, \mathrm{P}_{2}, \mathrm{P}_{3}$, and of the shoulder $\mathrm{P}_{4}$ are indicated by dashed vertical lines. Comparison of the VDOS in (a), (b) with (c) reveals distinct differences between the VDOS of the AF and FM state.

In Fig. 9(a), we present the experimental Fe-projected (partial) VDOS of the FM sample $\mathrm{Fe}_{51} \mathrm{Rh}_{49}$ (sample code FeRh02) obtained by NRIXS at $303 \mathrm{~K}$. For better comparison, in Fig. 9(b), we display again the experimental partial VDOS of the AF sample $\mathrm{Fe}_{48} \mathrm{Rh}_{52}$ at $305 \mathrm{~K}$, taken from Fig. 8(b). (A plot of the VDOS of $\mathrm{FM} \mathrm{Fe}_{51} \mathrm{Rh}_{49}$ at 63 and $303 \mathrm{~K}$ and of $\mathrm{AF}$ $\mathrm{Fe}_{48} \mathrm{Rh}_{52}$ at 59 and $305 \mathrm{~K}$ is presented in Fig. 7 of Ref. [77]). The VDOS of the FM and AF are clearly distinguishable. In fact, the features of the VDOS of the FM state, as shown in Fig. 9(a), are remarkably similar to those of the FM state above the AF-to-FM transition, [Fig. 8(c)], as described above. There is only a small red-shift $(\sim 2.2 \%$ averaged over the phonon spectrum) between the experimental FM VDOS in Fig. 9(a) (at $303 \mathrm{~K}$ ) and Fig. 8(c) (at $416 \mathrm{~K}$ ) due to lattice thermal expansion, otherwise both VDOS have nearly the same shape (a direct comparison of the VDOS of FM states, one for $\mathrm{Fe}_{51} \mathrm{Rh}_{49}$ at $303 \mathrm{~K}$ and the other one for $\mathrm{Fe}_{48} \mathrm{Rh}_{52}$ at $416 \mathrm{~K}$, 


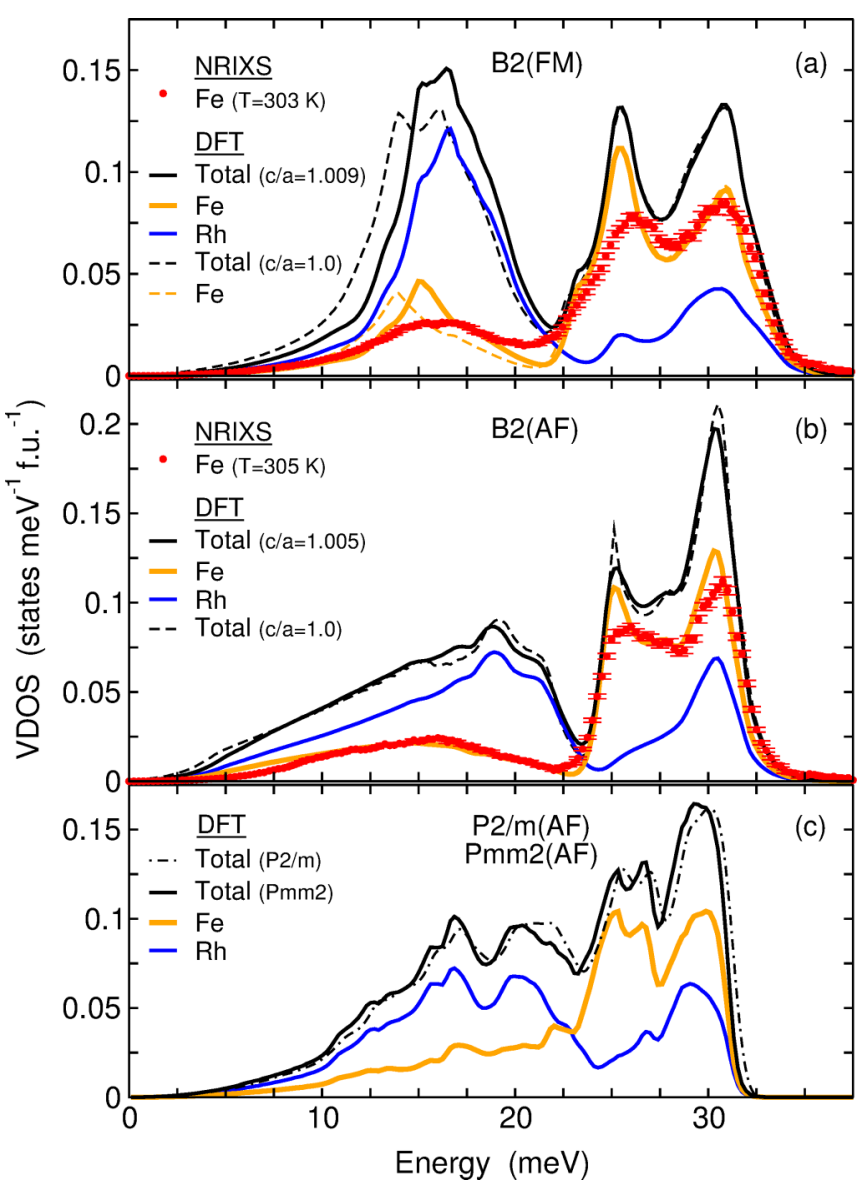

FIG. 9. Experimental Fe-projected VDOS (red circles with error bars) of $\mathrm{FM} \mathrm{Fe}_{51} \mathrm{Rh}_{49}$ measured by NRIXS at $303 \mathrm{~K}$ (a) and of AF $\mathrm{Fe}_{48} \mathrm{Rh}_{52}$ measured by NRIXS at $305 \mathrm{~K}$ (b) compared with the element-resolved (orange for Fe, blue for $\mathrm{Rh}$ ) and total (black) VDOS calculated from DFT at the respective experimental lattice constants (thick solid lines). This leads to slighly tetragonal cells $(c / a=1.009$ for FM and $c / a=1.005$ for AF according to the expitaxial strain), which we compare to cubic systems $(c / a=1.0)$ calculated at the respective atomic volume (dashed lines). This reveals a significant shift of the low-energy peak at $\sim 15 \mathrm{meV}$ on $c / a$. The lower panel (c) shows the total VDOS of the monoclinic $P 2 / m$ AF ground-state (dash-dotted black line, see Fig. 2 of Ref. [77] for the dispersion) together with the total and element resolved VDOS (thick black lines, same colors as above) for the orthorhombic Pmm2. Both structures yield very similar results which differ significantly from the measured VDOS in subfigures (a) and (b). All VDOS curves are specified in states per degree of freedom, $\mathrm{meV}$ and f.u. (formula unit or element) of stoichiometric FeRh.

is presented in Fig. 8 of Ref. [77]). The peak positions of the FM VDOS at $63 \mathrm{~K}$ are located at $\mathrm{P}_{1}=(31.4 \pm 0.2) \mathrm{meV}$, $\mathrm{P}_{2}=(26.2 \pm 0.3) \mathrm{meV}$, and $\mathrm{P}_{3}=(16.6 \pm 0.5) \mathrm{meV}$, and thus agree with the corresponding peak positions of the AF VDOS at low temperature $(59 \mathrm{~K})$ [Fig. 8(a)]. Besides the difference in peak heights, $P_{1}$ and $P_{2}$ becomes broader in the FM state than in the AF state (Fig. 9), the reverse is observed for $\mathrm{P}_{3}$ and $\mathrm{P}_{4}$.

It is important to compare the prominent features in our experimental Fe-projected VDOS of $\mathrm{AF} \mathrm{Fe}{ }_{48} \mathrm{Rh}_{52}$ with those of the [111] phonon dispersion obtained almost 40 years ago by inelastic neutron scattering on B2-ordered bulk FeRh at RT [58]. From the extrema (minima or maxima) observed in the [111] dispersion curve one would expect to find van-Hove-type anomalies in the corresponding VDOS. From the phonon dispersion in Fig. 3 of Ref. [58], we find a maximum at $\sim 31 \mathrm{meV}$ and a minimum at $\sim 26 \mathrm{meV}$ for the optical phonon modes, and a maximum at $\sim 24 \mathrm{meV}$ and a minimum at $\sim 17 \mathrm{meV}$ for the longitudinal acoustic phonon mode (we used the conversion $1 \mathrm{THz}=4.132 \mathrm{meV}$ ). The observed peak positions in the partial VDOS of our $\mathrm{AF} \mathrm{Fe}_{48} \mathrm{Rh}_{52}$ sample [Fig. 8(a)] are $31.0 \mathrm{meV}\left(\mathrm{P}_{1}\right), 25.5 \mathrm{meV}\left(\mathrm{P}_{2}\right)$, and $16.3 \mathrm{meV}\left(\mathrm{P}_{3}\right)$. A comparison shows that the latter peaks agree reasonably well with the position of extrema in the [111] phonon dispersion. This allows us to assign our peak $\mathrm{P}_{1}$ and $\mathrm{P}_{2}$ to the transverse and longitudinal optical mode, respectively, and $\mathrm{P}_{3}$ to the longitudinal acoustic mode. The VDOS-shoulder at $\sim 10 \mathrm{meV}$ has no counterpart in the [111] dispersion curve.

We find excellent agreement between the position of peaks in the experimental (NRIXS) VDOS and the positions of van Hove singularities expected from the phonon dispersion relations in Fig. 2 computed at the respective equilibrium volume. For the $\mathrm{AF}$ phase, we expect computed van Hove singularities at 10.2 $\pm 2.9,17.5 \pm 1.8,24.1 \pm 0.5$, and $29.2 \pm$ $1.1 \mathrm{meV}$, as compared with our experimental Fe-projected VDOS peaks at $10.0 \pm 1.5 \mathrm{meV}\left(\mathrm{P}_{4}\right), 16.3 \pm 0.5 \mathrm{meV}\left(\mathrm{P}_{3}\right)$, $25.5 \pm 0.3 \mathrm{meV}\left(\mathrm{P}_{2}\right)$, and $31.0 \pm 0.2 \mathrm{meV}\left(\mathrm{P}_{1}\right)$. The agreement is remarkable in view of the fact that no adjustable parameters were employed. The same is valid for the FM phase: we expect computed van Hove singularities at $14.1 \pm 1.9,24.9 \pm 1.8$, and $30.4 \pm 0.6 \mathrm{meV}$, in comparison with $16.6 \pm 0.5 \mathrm{meV}$ $\left(\mathrm{P}_{3}\right), 26.2 \pm 0.3 \mathrm{meV}\left(\mathrm{P}_{2}\right)$, and $31.4 \pm 0.2 \mathrm{meV}\left(\mathrm{P}_{1}\right)$ in the experiment. The remaining disagreement is remedied if the VDOS is compared to a calculation carried out at the experimental lattice parameters as shown in Fig. 9. This applies in particular to Peak $\mathrm{P}_{3}$ in the $\mathrm{B} 2(\mathrm{FM})$ phase, for which we obtain $14.1 \pm 1.9 \mathrm{meV}$ from Fig. 2 versus $(16.6 \pm 0.5) \mathrm{meV}$ in the experiment. In the latter case, the epitaxial condition implies a slight tetragonal distortion. This indeed causes a significant shift $(1.5 \mathrm{meV})$ of $\mathrm{P}_{3}$ to higher energies, which is seen by comparing the cubic FM VDOS (dashed yellow lines) to the tetragonal distorted one (solid yellow lines) at the same volume per atom [Fig. 9(a)]. From the qualitative difference between the experimental AF-VDOS in Fig. 9(b) and the calculated results for the predicted new AF ground-state structures shown in Fig. 9(c), we infer that no significant fraction of $P 2 / m$ and $P m m 2$ structures is present in the experimental samples down to at least $60 \mathrm{~K}$. This becomes evident from the deep minimum between $\mathrm{P}_{2}$ and $\mathrm{P}_{3}$ in the $\mathrm{B} 2(\mathrm{AF})$ phase at $23 \mathrm{meV}$, which is fully reproduced by the experiment, while the computational VDOS of the monoclinic and orthorhombic structures is larger by one order of magnitude.

Recently, anomalous structural behavior across the metamagnetic transition of $\mathrm{Fe}_{49} \mathrm{Rh}_{51}$ thin films on $\mathrm{MgO}(001)$ has been observed by temperature-dependent extended $\mathrm{x}$-ray absorption fine structure (EXAFS) studies [59]. The authors extracted the $T$ dependence of the EXAFS dynamical Debye-Waller factor (or mean-square relative atomic displacement $C_{2}=$ $\left.\left\langle(r-\langle r\rangle)^{2}\right\rangle\right)$ of $\mathrm{Fe}-\mathrm{Rh}, \mathrm{Fe}-\mathrm{Fe}$, and Rh-Rh from the $\mathrm{Fe}$ and Rh $K$-edge EXAFS signals. Anomalous thermal behavior near the AF to FM transition (including a thermal hysteresis) 
was observed in particular in $C_{2}$ for $\mathrm{Fe}-\mathrm{Fe}$ and $\mathrm{Rh}-\mathrm{Rh}$, but less so for Rh-Fe and Fe-Rh. As NRIXS measures the $T$ dependence of the total $\mathrm{Fe}$ mean-square displacement, $\left\langle x^{2}\right\rangle$, it is interesting to compare our NRIXS $\left\langle x^{2}\right\rangle$ values with the EXAFS $C_{2}$ data in Fig. 5 of Ref. [59]. We observe a tendency for our $\left\langle x^{2}\right\rangle$ data to be slightly lower for the FM phase than for the AF state at corresponding temperatures. Compared to the EXAFS $C_{2}$ values (as plotted in Fig. 5 of Ref. [59]) our $\mathrm{Fe}\left\langle x^{2}\right\rangle$ NRIXS data (given in detail in Table II of Ref. [77]) are close to the $C_{2}$ values for $\mathrm{Fe}-\mathrm{Rh}$ vibrations, but distinctly different to the $C_{2}$ values for $\mathrm{Fe}-\mathrm{Fe}$ vibrations. This observation suggests that the dominant contribution to the NRIXS $\left\langle x^{2}\right\rangle$ originates from nearest-neighbor $\mathrm{Fe}-\mathrm{Rh}$ vibrational modes. On the other hand, the larger differences in the $\mathrm{Fe}-\mathrm{Fe}$ and $\mathrm{Rh}-\mathrm{Rh}$ Debye-Waller factors compared to the mixed case reported by EXAFS indicates that in the AF phase vibrations are enhanced in the planes formed by either $\mathrm{Fe}$ or $\mathrm{Rh}$ alone. This could be a manifestation of the soft mode at $X$ as described in Sec. III A, which is present in the AF but not in the FM phase (see also the inset of Fig. 4).

From the experimental VDOS, $g(E)$, we obtain the Feprojected lattice entropy $S_{\mathrm{Fe}}^{\mathrm{vib}}\left(M_{\exp }, V_{\exp }\right)$ and lattice specific heat $C_{\mathrm{Fe}}^{\mathrm{vib}}\left(M_{\exp }, V_{\exp }\right)$ corresponding to constant volume $V_{\exp }$ and magnetization $M_{\exp }$ at the respective measurement temperature $T_{\exp }$ [113]. The experimental difference $\Delta C^{\mathrm{vib}}(\mathrm{Fe})=C_{\mathrm{FM}}^{\mathrm{vib}}(\mathrm{Fe})-C_{\mathrm{AF}}^{\text {vib }}(\mathrm{Fe})$ between the $\mathrm{FM}$ and $\mathrm{AF}$ states in the measured temperature range is very small. Near $60 \mathrm{~K}$, the difference is found to be $\Delta C^{\mathrm{vib}}(\mathrm{Fe})=[0.701(2)-$ $0.645(2)] k_{\mathrm{B}} / \mathrm{Fe}=+0.056(3) k_{\mathrm{B}} / \mathrm{Fe}$, i.e., $C_{\mathrm{FM}}^{\mathrm{vib}}(\mathrm{Fe})>C_{\mathrm{AF}}^{\mathrm{vib}}(\mathrm{Fe})$. At room temperature, we find $\Delta C^{\mathrm{vib}}(\mathrm{Fe})=[2.757(8)-$ $2.765(7)] k_{\mathrm{B}} / \mathrm{Fe}=-0.008(10) k_{\mathrm{B}} / \mathrm{Fe}$, being zero within error bars. Also the difference obtained from DFT, $\Delta C^{\mathrm{vib}}(\mathrm{Fe})=$ $-0.003 k_{\mathrm{B}} / \mathrm{Fe}$, is very small.

Likewise, the difference $\Delta S^{\mathrm{vib}}(\mathrm{Fe})=S_{\mathrm{FM}}^{\mathrm{vib}}(\mathrm{Fe})-S_{\mathrm{AF}}^{\mathrm{vib}}(\mathrm{Fe})$ between the FM and AF states in the measured temperature range is found to be small and changes sign. Near $60 \mathrm{~K}$, we obtain $\Delta S^{\mathrm{vib}}(\mathrm{Fe})=[0.261(1)-0.254(1)] k_{\mathrm{B}} / \mathrm{Fe}=$ $0.0070(14) k_{\mathrm{B}} / \mathrm{Fe}$, with $S_{\mathrm{FM}}^{\text {vib }}(\mathrm{Fe})>S_{\mathrm{AF}}^{\text {vib }}(\mathrm{Fe})$. At RT, for example, we find that $\Delta S^{\mathrm{vib}}(\mathrm{Fe})=[3.345(9)-3.410(8)] k_{\mathrm{B}} / \mathrm{Fe}=$ $-0.065(12) k_{\mathrm{B}} / \mathrm{Fe}$, with $S_{\mathrm{FM}}^{\text {vib }}(\mathrm{Fe})<S_{\mathrm{AF}}^{\text {vib }}(\mathrm{Fe})$, which is consistent with the increased mean square displacements in the AF phase observed by both NRIXS here and EXAFS in Ref. [59].

From our calculations, we obtain a somewhat larger absolute value of $\Delta S^{\mathrm{vib}}(\mathrm{Fe})=-0.114 k_{\mathrm{B}} / \mathrm{Fe}$ at RT. For the joint contribution of both elements to the entropy change our calculations yield $\Delta S^{\mathrm{vib}}=-0.210 k_{\mathrm{B}} /$ f.u., which increases significantly to $\Delta S^{\text {vib }}=-0.058 k_{\mathrm{B}} /$ f.u., if we calculate it for the corresponding cubic systems at the same atomic volume. ${ }^{3}$ Since the VDOS of the AF phase remains essentially unchanged by the tetragonal distortion, this difference is

\footnotetext{
${ }^{3}$ The unit $k_{\mathrm{B}} / \mathrm{Fe}$ multiplied by the factor 8.480 (for $\mathrm{Fe}_{51} \mathrm{Rh}_{49}, 95 \%$ enriched in $\left.{ }^{57} \mathrm{Fe}\right)$ yields the unit $\mathrm{J} \mathrm{K}^{-1}\left(\text { mole } \mathrm{Fe}_{51} \mathrm{Rh}_{49}\right)^{-1}$. The unit $k_{\mathrm{B}} / \mathrm{Fe}$ multiplied by the factor 7.982 (for $\mathrm{Fe}_{48} \mathrm{Rh}_{52}, 95 \%$ enriched in $\left.{ }^{57} \mathrm{Fe}\right)$ yields the unit $\mathrm{J} \mathrm{K}{ }^{-1}\left(\text { mole } \mathrm{Fe}_{48} \mathrm{Rh}_{52}\right)^{-1}$. The unit $k_{\mathrm{B}} / \mathrm{Fe}$ multiplied by the factor 53.356 (for $\mathrm{Fe}_{51} \mathrm{Rh}_{49}, 95 \%$ enriched in ${ }^{57} \mathrm{Fe}$ ) yields the unit $\mathrm{J} \mathrm{K}^{-1} \mathrm{~kg}^{-1}$, and the unit $k_{\mathrm{B}} / \mathrm{Fe}$ multiplied by the factor 49.369 (for $\mathrm{Fe}_{48} \mathrm{Rh}_{52}, 95 \%$ enriched in ${ }^{57} \mathrm{Fe}$ ) yields the unit $\mathrm{J} \mathrm{K}^{-1} \mathrm{~kg}^{-1}$. $1 \mathrm{~mol}$ of ${ }^{57} \mathrm{Fe}$-enriched $\mathrm{Fe}_{51} \mathrm{Rh}_{49}\left(\mathrm{Fe}_{48} \mathrm{Rh}_{52}\right)$ corresponds to $158.93 \mathrm{~g}$
}

predominantely caused by the shift of the peak $\mathrm{P}_{3}$ in the FM phase. It is not advisable to compare these values directly with the total entropy change from bulk experiments, since the different composition of the films affect the lattice parameters and thus decrease the volume change at the phase transition, which, according to Grüneisen theory, has a considerable impact on the entropy change. We may, however, compare the entropy change with and without tetragonal distortion, since the volume of each phase is kept constant. From the values given above for $\Delta S^{\mathrm{vib}}$, we might expect an increase of the transition temperature by $\Delta T \approx T\left(\Delta S^{\text {tetra }}-\Delta S^{\text {cubic }}\right) / C_{p} \approx$ $8 \mathrm{~K}$ from the impact of a tetragonal distortion of less than $1 \%$ on the vibrational entropy of the FM phase. This shift supports the recently reported isothermal metamagnetic transition in $\mathrm{FeRh} / \mathrm{BaTiO}_{3}$ heterostuctures through electric-field induced tetragonal strain [19]. Uniaxial strain conditions might also evolve in bulk systems from the large volumetric stress during the transformation. This possibly contributes to the rather large hysteresis associated with the metamagnetic transition. In turn, it has been shown recently, that multistimuli cycles [29] combining the magnetocaloric transition with biaxial compressive strain can effectively decrease hysteresis losses [36].

\section{THERMODYNAMIC STABILITY FROM QUASIHARMONIC CALCULATIONS}

To quantify the relevance of the lattice and electronic degrees of freedom for the thermodynamic stability of the $\mathrm{B} 2(\mathrm{FM})$, the $\mathrm{B} 2(\mathrm{AF})$, and the new hypothetical lowtemperature phase, we calculate the free energies from firstprinciples within the quasiharmonic approximation [113-115] and derived $V(T), C_{p}(T)$, and $S(T)$, which can be directly compared with experiments. We approximate the monoclinic low-temperature structure by an orthorhombic model (point group $P \mathrm{~mm} 2$ ). This saves significant computation time since the unit cell lacks the small monoclinic distortion, but still exhibits a stable phonon dispersion. It also has a closely related VDOS to the $P 2 / \mathrm{m}$ ground state and is extremely close in energy (+1.6 meV/f.u.).

Minimization of the free energy $F=E-T S$ with respect to the volume $V$ at a given temperature $T$ yields the Gibbs free energy $G$ at zero pressure. Magnetic contributions to the Gibbs free energy are not included here. The corresponding thermal expansion arises from the volume dependence of the vibrational and electronic DOS. We obtain a similar temperature dependence in all three phases with a linear thermal expansion coefficient at room temperature of $1.3 \times$ $10^{-5} \mathrm{~K}^{-1}$ for $\mathrm{B} 2(\mathrm{AF})$ and $1.1 \times 10^{-5} \mathrm{~K}^{-1}$ for $\mathrm{B} 2(\mathrm{FM})$ and Pmm2, which corresponds reasonably well to the value of $0.95 \times 10^{-5} \mathrm{~K}^{-1}$ obtained by Ibarra and Algabarel for the AF phase of FeRh [86] (see also Fig. 9 in Ref. [77]). The calculated volume change between $\mathrm{B} 2(\mathrm{AF})$ and $\mathrm{B} 2(\mathrm{FM})$ of $1.5 \%$ at $T=350 \mathrm{~K}$ slightly exceeds the experimental reports of around $1 \%[116,117]$.

(161.69 g). For comparison, the conversion factor between $k_{\mathrm{B}} /$ f.u. ( 1 f.u. $=2$ atoms) or $k_{\mathrm{B}} /$ element and $\mathrm{J} \mathrm{K}^{-1} \mathrm{~kg}^{-1}$ of natural stoichiometric FeRh amounts to 52.371. 


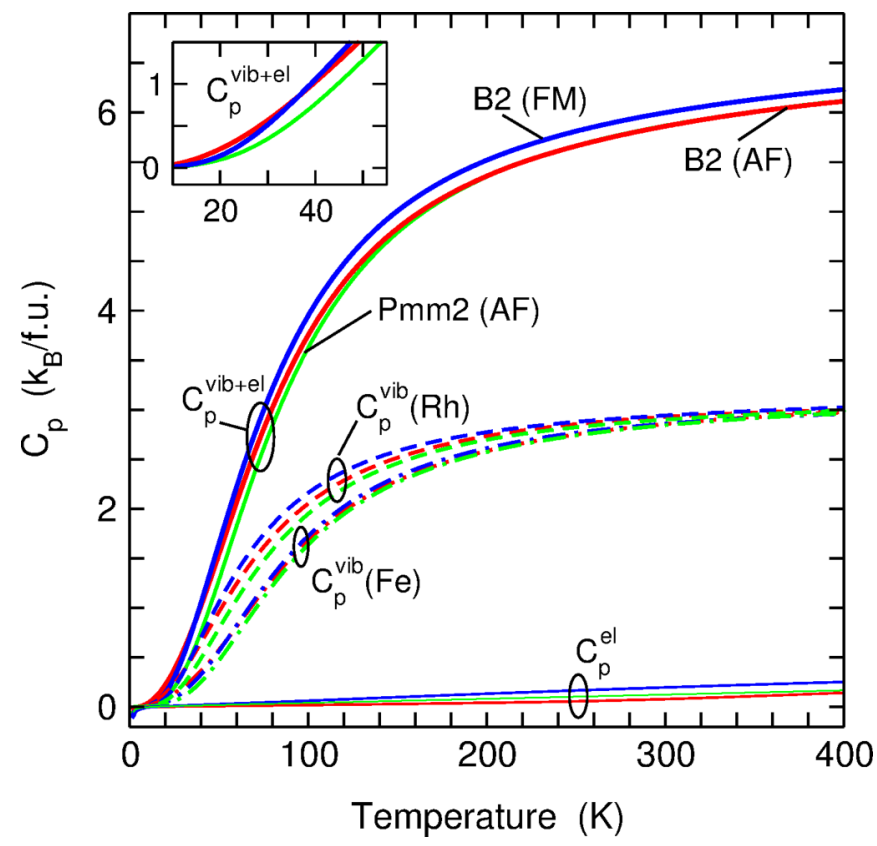

FIG. 10. Lattice and electronic specific heat $C_{p}$ at zero pressure for the B2(AF) (red), the B2(FM) (blue), and the orthorhombic Pmm2(AF) (green) FeRh structures. The plot shows the sum of electronic and lattice specific heat (thick solid lines) and the element-resolved contributions to the vibrational degrees of freedom (dashed lines for $\mathrm{Rh}$ and dash-dotted lines for $\mathrm{Fe}$ ). The contributions from $\mathrm{Rh}$ are larger than from $\mathrm{Fe}$. The excess in $C_{p}$ of the FM state at room temperature and above is entirely related to the electronic contribution (thin solid lines). Below $T=200 \mathrm{~K}$, there is also a notable contribution from the lattice degrees of freedom corresponding to $\mathrm{Rh}$. The inset shows the crossover in $C_{p}^{\mathrm{vib}+\mathrm{el}}$ of $\mathrm{B} 2(\mathrm{AF})$ and $\mathrm{B} 2(\mathrm{FM})$ at low temperatures.

Knowledge of $V(T)$ yields access to $S(T)$ and the specific heat at constant pressure $C_{p}^{\mathrm{vib}+\mathrm{el}}=T(\partial S / \partial T)_{p}$ arising from the electronic and lattice degrees of freedom. This quantity is shown in Fig. 10, further decomposed into the electronic and element specific vibrational contributions, $C_{p}^{\mathrm{el}}$ and $C_{p}^{\mathrm{vib}}$, respectively. We see that from $T=40 \mathrm{~K}$ upwards the FM phase exhibits a significantly larger $C_{p}^{\mathrm{vib}+\mathrm{el}}$ than the other two phases. Below $T=200 \mathrm{~K}$, this is caused by the larger lattice specific heat of Rh, $C_{p}^{\mathrm{vib}}(\mathrm{Rh})$. Since the motion of the lighter element $\mathrm{Fe}$ is, as expected, represented by the higher phonon frequencies, we find $C_{p}^{\mathrm{vib}}(\mathrm{Fe})<C_{p}^{\mathrm{vib}}(\mathrm{Rh})$ for all $T$. The contribution of $\mathrm{Fe}$ is approximately the same for all three phases. Above room temperature, where the vibrational specific heat at constant $V$ approaches the Dulong-Petit limit, the difference between the phases in $C_{p}^{\mathrm{vib}+\mathrm{el}}$ is dominated by the difference between the electronic contributions, which is largest for the FM phase according to the larger electronic DOS at $E_{\mathrm{Fermi}}$. This corroborates that the electronic degrees of freedom deliver an important contribution to the thermodynamic stability at elevated temperatures, as was suggested earlier based on experimental work [42] and confirmed later from DFT calculations [53-55,107,118]. Below $T=40 \mathrm{~K}$, the lattice specific heat of the $\mathrm{B} 2(\mathrm{AF})$ phase exceeds the contribution of the B2(FM) phase (inset of Fig. 10). The inversion at low temperatures was predicted in the early phenomenological

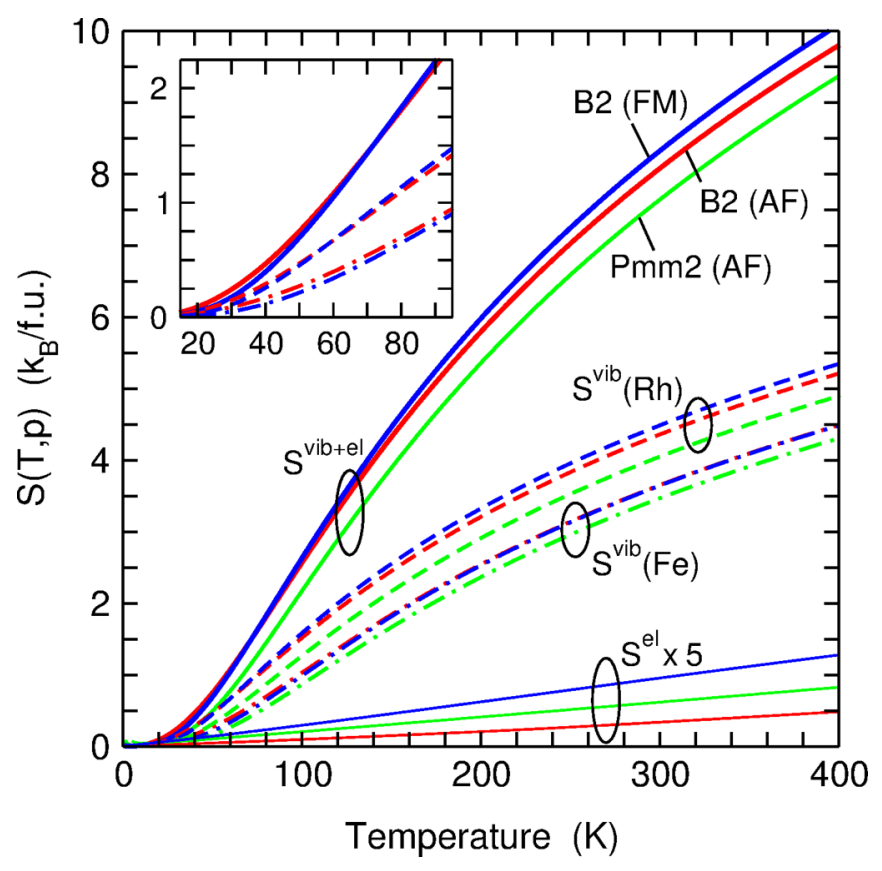

FIG. 11. Electronic and element-resolved contributions from the lattice degrees of freedom to the entropy $S(T, p)$ at zero pressure for B2-FeRh (AF and FM) and the Pmm 2 structure. Colors and line patterns correspond to Fig. 10. Around room temperature the sum of the lattice and electronic entropy $S^{\mathrm{vib}+\mathrm{el}}$ of the FM phase exceeds the entropy of the AF B2-phase. This difference is mainly related to the electronic entropy $S^{\text {el }}$ (thin solid lines, enlarged by a factor of 5) and the contribution from the lattice entropy associated with $\mathrm{Rh}$ (dahes lines), originating from the excess specific heat of the FM phase around $100 \mathrm{~K}$ visible in Fig. 10. The inset diplays the low-temperature behavior of the total and lattice entropy. The vibrational entropy of Fe nearly coincides for B2(AF) and B2(FM).

analysis of Ricodeau and Melville [119] and later observed in the thin film experiments of Cooke and coworkers [46]. Such a crossover can be inferred from the linear coefficient of the specific heat $\gamma$ which is significantly larger for the FM phase according to the larger electronic density of states (cf. Fig. 6). From a fit to our electronic entropy data below $100 \mathrm{~K}$, we obtain $\gamma_{\mathrm{B} 2(\mathrm{AF})}=3.84 \times 10^{-5} k_{\mathrm{B}} \mathrm{K}^{-1}$ f.u. $^{-1}=2.01 \mathrm{~mJ} \mathrm{~kg}^{-1} \mathrm{~K}^{-2}$, $\gamma_{\mathrm{B} 2 \text { (FM) }}=1.17 \times 10^{-4} k_{\mathrm{B}} \mathrm{K}^{-1}$ f.u. $^{-1}=6.10 \mathrm{~mJ} \mathrm{~kg}^{-1} \mathrm{~K}^{-2}$, and $\gamma_{P m m 2(\mathrm{AF})}=8.36 \times 10^{-5} k_{\mathrm{B}} \mathrm{K}^{-1}$ f.u. ${ }^{-1}=4.38 \mathrm{~mJ} \mathrm{~kg}^{-1} \mathrm{~K}^{-2}$, for the B2(AF), the B2(FM) and the Pmm2 configurations, respectively. These values are in good agreement with previous theoretical and experimental estimates [42-44,46], but too small to account for the magnitude of the effect shown in the inset of Fig. 10, which we rather relate to the excitation of the low-lying soft phonon branches of the B2(AF) phase.

The larger $C_{p}^{\text {vib+el }}$ of $\mathrm{B} 2(\mathrm{FM})$ in a wide temperature range corresponds to a larger entropy $S^{\mathrm{vib}+\mathrm{el}}$ for $T>70 \mathrm{~K}$. Keeping in mind the discussion of $C_{p}$, this originates from the larger vibrational contribution of $\mathrm{Rh}$ in combination with the electronic entropy, which steadily increases with temperature. At low temperatures, $S^{\mathrm{vib}+\mathrm{el}}$ is dominated by the low-lying phononic modes in the $\mathrm{B} 2(\mathrm{AF})$ phase and exceeds the entropy in the FM phase below $70 \mathrm{~K}$, as shown in the inset of Fig. 11. 
At $T=350 \mathrm{~K}$, which corresponds to the metamagnetic transition, we obtain a considerable difference in entropy of $\Delta S^{\mathrm{vib}+\mathrm{el}}=S_{\mathrm{B} 2(\mathrm{FM})}^{\mathrm{vib}+\mathrm{el}}-S_{\mathrm{B} 2(\mathrm{AF})}^{\mathrm{vib}+\mathrm{el}}=0.268 k_{\mathrm{B}} / \mathrm{f} . \mathrm{u} .=$ $11.7 \mathrm{~J} \mathrm{~kg}^{-1} \mathrm{~K}^{-1}$, which is close to the experimentally reported values of the total entropy change obtained in field-, pressure-, and temperature-induced transitions, ranging from $12 \mathrm{~J} \mathrm{~kg}^{-1} \mathrm{~K}^{-1}$ to $19 \mathrm{~J} \mathrm{~kg}^{-1} \mathrm{~K}^{-1}[26,30,33,38,56,57,116]$. Our $\Delta S^{\mathrm{vib}+\mathrm{el}}$ is a sum of nearly equal parts of the electronic entropy and the lattice contribution of Rh. The vibrational degrees of freedom of $\mathrm{Fe}$ apparently do not contribute to the difference in entropy between B2(FM) and B2(AF) in the relevant temperature range. Their contribution might even be slightly negative, since the quasiharmonic calculations overestimate the volume change at the phase transition by $0.5 \%$. Our computational results are thus not conflicting with our experimental measurements, which clearly show $S_{\mathrm{AF}}^{\mathrm{vib}}(\mathrm{Fe})>S_{\mathrm{FM}}^{\mathrm{vib}}(\mathrm{Fe})$ at $\mathrm{RT}$, as this can be traced back to the tetragonal distortion of the films in combination with a smaller volume difference (cf. Sec. IV), which is about half of the reported value for bulk FeRh.

Comparing $\Delta S^{\mathrm{vib}+\mathrm{el}}$ with the earlier theoretical estimates for $\Delta S^{\text {mag }}$ ranging between $6 \mathrm{~J} \mathrm{~kg}^{-1} \mathrm{~K}^{-1}, 8 \mathrm{~J} \mathrm{~kg}^{-1} \mathrm{~K}^{-1}$ (Refs. [45] and [49], from empirical spin-model calculations), and $14 \mathrm{~J} \mathrm{~kg}^{-1} \mathrm{~K}^{-1}$ (Ref. [55], from the magnon density of states), we conclude that lattice, electronic, and magnetic degrees of freedom contribute in roughly equal magnitude at the metamagnetic transition.

The observation that the Pmm2 structure has the lowest specific heat for $T<250 \mathrm{~K}$ is reflected in a significantly lower entropy compared to the B2 phases in the entire temperature range (cf. Fig. 11). In relation to the $\mathrm{B} 2(\mathrm{AF})$ phase the $P m m 2(\mathrm{AF})$ phase, at $T=350 \mathrm{~K}$, has a rather large $\Delta S^{\mathrm{vib}+\mathrm{el}}=-0.434 k_{\mathrm{B}} /$ f.u. $=-22.7 \mathrm{~J} \mathrm{~kg}^{-1} \mathrm{~K}^{-1}$. Once again, this difference is dominated by the lattice contribution of $\mathrm{Rh}$ $\left(\Delta S^{\mathrm{vib}}(\mathrm{Rh})=-0.313 k_{\mathrm{B}}\right)$.

Figure 12 shows the different contributions to the Gibbs free energy difference of the Pmm 2(AF) and B2(FM) phases with respect to the $\mathrm{B} 2(\mathrm{AF})$ phase. In addition to the electronic and lattice part, we also show the quasiharmonic Gibbs free energy $G^{\text {gha }}(T)$, which includes all contributions, except for the magnetic part which has not been calculated in this work and the DFT ground-state total energy $E(V(0))$, which turns out to be particularly sensitive to methodological details:

$$
G^{\mathrm{gha}}(T)=G^{\mathrm{vib}}(T)+G^{\mathrm{el}}(T)+E(V(T))-E(V(0)) .
$$

According to the large $\Delta S^{\mathrm{vib}}$, the difference in the lattice contributions $\Delta G^{\mathrm{vib}}(T)$ between Pmm2(AF) and $\mathrm{B} 2(\mathrm{AF})$ is steeply increasing with temperature. Thus the hypothetical low-temperature structure is suppressed with respect to the $\mathrm{B} 2(\mathrm{AF})$ phase by lattice entropy, providing a further reason for the absence of experimental indications for a new ground state, including our NRIXS ${ }^{57} \mathrm{Fe}-\mathrm{VDOS}$ in Fig. 8(a).

Concerning the isostructural metamagnetic transition, we observe a clear preference for the FM phase, which at ambient conditions, still arises in large parts from the difference in the zero-point energies and the free energy of the electronic system. The magnitude of $\Delta G^{\mathrm{el}}$ at room temperature corre-

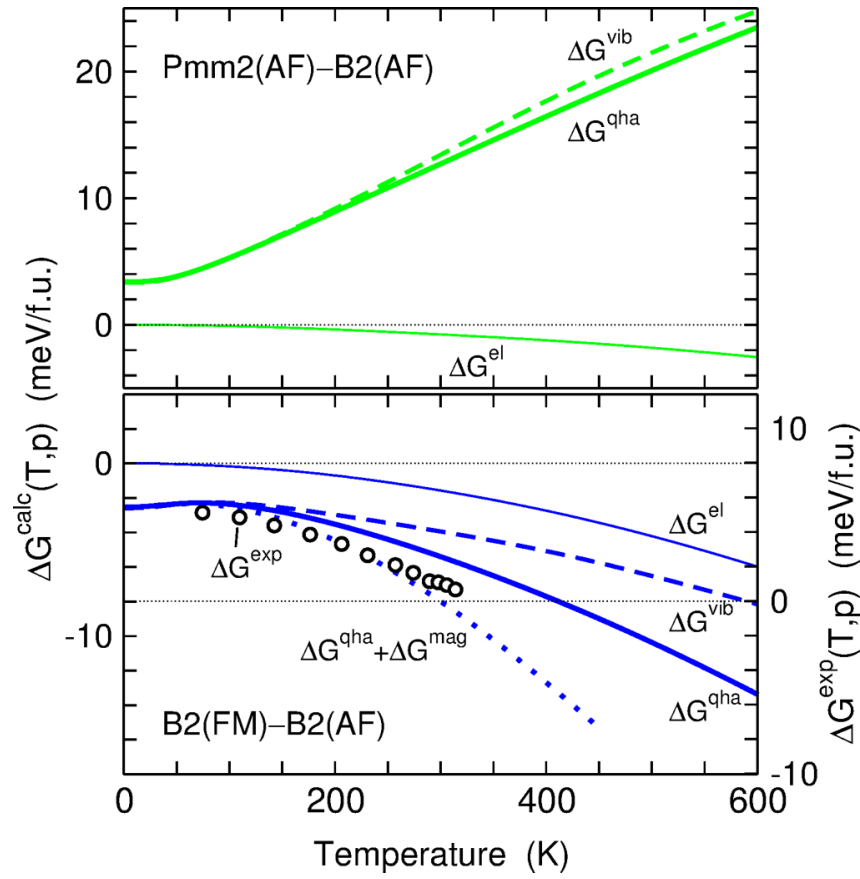

FIG. 12. (Left axis) Contributions to the calculated Gibbs free energy difference $\Delta G^{\text {calc }}$ of the orthorhombic $P m m 2$ structure (upper panel) and the cubic B2(FM) phase (lower panel) relative to the $\mathrm{B} 2(\mathrm{AF})$ phase as a function of temperature. Thick lines refer to the quasiharmonic contribution $\Delta G^{\text {qha }}$ according to Eq. (1), thin solid lines to the electronic part $\Delta G^{\mathrm{el}}$ and dashed lines to the pure vibrational contribution $\Delta G^{\mathrm{vib}}$. The dotted line in the lower panel denotes the sum of $\Delta G^{\text {qha }}$ and the estimate of the magnetic Gibbs free energy difference $\Delta G^{\mathrm{mag}}$ calculated by Gu and Antropov [55] from the magnon density of states. The open circles show the experimental Gibbs free energy differences $\Delta G^{\text {exp }}$ and refer to the right axis. The scales of both axes are matched by an appropriate offset $\Delta G^{\exp }(0)-\Delta G^{\mathrm{vib}}(0)=7.97 \mathrm{meV} /$ f.u. such that experimental and theoretical data sets may be directly compared.

sponds well to the estimate of Déak et al. [53], which takes into account the changes to the electronic structure around $E_{\mathrm{Fermi}}$ at finite temperatures through magnetic excitation in the framework of the disordered local moment (DLM) approach. Combining $\Delta G^{\text {qha }}$ with the ground-state energy difference $\Delta E^{\mathrm{DFT}}$ from Table $\mathrm{I}$, this quantity turns out to be almost one order of magnitude too small to account for the experimental phase transition temperature. This mismatch does not disappear if we take into account the magnetic Gibbs free energy difference $\Delta G^{\mathrm{mag}}$ calculated by Gu and Antropov [55] from the magnon dispersion relations obtained with DFT calculations. Keeping in mind the good agreement of the magnitude of $\Delta S^{\mathrm{vib}+\mathrm{el}}$ with the experimental entropy change, it is clear that the ground-state energy differences between $\mathrm{B} 2(\mathrm{AF})$ and $\mathrm{B} 2(\mathrm{FM})$ from our semilocal DFT calculations, grossly overestimate the $T=0$ free energy difference between the two phases. This applies as well to the vast majority of DFT investigations although a recent comparison of the electronic density of states obtained from DFT calculations and HAXPES measurements[120] confirms a proper description 
of the electronic structure within the standard semilocal GGA used in our approach. ${ }^{4}$

Also the early experiment of Ponomarev [57] yields a much smaller estimate for the Gibbs free energy difference at $T=$ 0 of $\Delta G^{\exp }(0)=3.23 \times 10^{-10} \mathrm{~J} / \mathrm{kg}=5.38 \mathrm{meV} /$ f.u. for a $\mathrm{Fe}_{0.96} \mathrm{Rh}_{1.04}$ alloy, which was confirmed in its magnitude from the specific heat measurements of Cooke and coworkers [46]. In contrast to $\Delta E^{\mathrm{DFT}}, \Delta G^{\mathrm{exp}}(0)$ already includes the zero point energies of the phonons of both phases as well as a possible zero point contribution of the antiferromagnetic magnons predicted from spin-wave theory [123-125]. As pointed out recently by Polesya $e t$ al. by means of DLM calculations, the energy difference between the FM and AF structure depends decisively on the magnetic order and changes its sign when the magnetic order parameter drops below a critical value of 0.8 [54]. On the other hand, in their thermodynamic analysis of magnetic and electronic free energies, which includes spin disorder but neglects lattice contributions, Déak et al. [53] did not encounter a transition for a volume below $30.08 \AA^{3} /$ f.u., which exceeds the experimental volume range.

The data points from Ref. [57] coincide well with the sum of $\Delta G^{\text {qha }}(T)$ and the magnetic contribution $\Delta G^{\mathrm{mag}}(T)$, as calculated by $\mathrm{Gu}$ and Antropov from the magnon density of states [55]. Combining $\Delta G^{\text {qha }}(T)$ with $\Delta G^{\exp }(0)$ taken from Ref. [57], we obtain a transition temperature of $T_{\mathrm{M}}^{\text {qha }}=410 \mathrm{~K}$. From the sum $\Delta G^{\mathrm{qha}}(T)+\Delta G^{\mathrm{mag}}(T)$ a value of $T_{\mathrm{M}}^{\text {qha+mag }}=$ $300 \mathrm{~K}$ is found, which should be compared to $T_{\mathrm{M}}^{\exp }=$ $331 \mathrm{~K}$, as reported by Ponomarev. Combining alternatively $\Delta G^{\text {qha }}(T)$ with the free energy contribution obtained from the empirical Blume-Capel model discussed in Ref. [45] yields $T_{\mathrm{M}}^{\exp }=350 \mathrm{~K}$ (see Sec. V of Ref. [77] for further details). Keeping in mind that we neglect anharmonic contributions and cross-coupling between the magnetic, electronic and vibrational degrees of freedom, we can rate this an excellent agreement, which corroborates our present decomposition of the thermodynamic quantities.

\section{CONCLUSIONS}

With our combined ab initio and experimental approach, we provide a comprehensive survey on the lattice dynamical properties of the AF and FM phases of B2 ordered FeRh and their relation to the metamagnetic transition. Our experimental NRIXS investigation of the partial Fe VDOS in the FM and $\mathrm{AF}$ phase is the first of its kind for FeRh and allows an independent experimental assessment of the element-resolved vibrational contributions to specific heat and entropy. The comparison with the element-resolved VDOS obtained from first-principles calculations yields a very good agreement for both phases. For the FM phase, the agreement is substantially improved with respect to a Rh-dominated peak around $15 \mathrm{meV}$ by considering the tiny tetragonal distortion of the thin film in the calculations. This reveals an unexpected strong sensitivity

\footnotetext{
${ }^{4}$ However, we note that there exist a few exceptions reporting a significantly smaller energy difference, as the aforementioned work of $\mathrm{Gu}$ and Antropov [55], which is based on the less common LangrethMehl functional [121] and likewise a recent GGA $+U$ approach [122] accounting for additional correlation on $\mathrm{Fe} d$ sites.
}

of the FM phonons on uniaxial strain, which is not present at all in the AF phase. Thus strain conditions must be considered explicitly in the comparison of the thermodynamic properties of the bulk material and epitaxial thin films. In particular, a Debye model fitted to bulk elastic parameters as used in Ref. [46] does not allow a sufficiently accurate decomposition of the specific heat and entropy change measured in thin films. The combination with $a b$ initio work as presented in this and other recent studies $[31,53,54]$ could in turn lead to a precise estimate for the magnetic specific heat.

In the $\mathrm{AF}$ phase, we encounter soft phonon branches along [110], which become imaginary in a small fraction of reciprocal space around the $X$ point, which is in agreement with another recent investigation [60]. The instability acts as a precursor for a competing monoclinic AF structure with lower energy. A possible origin of this instability are symmetry related changes in the electronic structure, which however, are most prominent far below the Fermi energy. Accordingly, the Fermi surface of B2-ordered FeRh in the AF phase is comparably small and shows no signs of apparent nesting. This is in contrast to the case of $\mathrm{L} 2{ }_{1}$ Heusler compounds like $\mathrm{Ni}_{2} \mathrm{MnGa}$, which are similar in structure and exhibit also a pronounced phonon instability along [110] as martensitic precursor.

For B2 FeRh, the energy gain on the calculated barrier free transformation pathway is only $0.2 \mathrm{meV} /$ f.u., so even small thermal fluctuations may suppress the transition to the monoclinic phase, although the fully distorted structure would be clearly favored in energy compared to the B2 phase. This could explain why we do not find any trace of the new phase down to a temperature of $59 \mathrm{~K}$ in our NRIXS VDOS measurements. This is in accordance with our first-principles estimate of the vibrational and electronic Gibbs free energy in the quasiharmonic approximation, which also suggests a strong entropic suppression of the new phase with increasing temperature.

On the other hand, the FM B2 structure is favored by lattice and electronic entropy. Together with the magnetic free energy calculated from the magnon dispersion relations taken from Ref. [55], we obtain an excellent agreement with the early measurement of Ponomarev [57], which is up to now the only experimental benchmark for the free energy. From the close agreement between theory and experiment for specific heat, entropy and Gibbs free energy, we conclude that our decomposition provides a realistic estimate of the impact of the different degrees of freedom on the metamagnetic transition: we propose a cooperative and essentially equal contribution of the magnetic and the combined electronic and vibrational degrees of freedom. The latter is associated mainly with $\mathrm{Rh}$, whereas the lattice contribution from $\mathrm{Fe}$ is small. The availability of detailed information on the thermodynamic contributions of the lattice and the electrons opens a new route to benchmark the different magnetic models and-once specific heat, entropy, and free energy data are made available - may settle the long-standing dispute about the origin of the metamagnetic transition.

\section{ACKNOWLEDGMENTS}

The authors would like to thank Sergii Khmelevskyi, Martijn Marsman, and Sebastian Fähler for fruitful discussions. We 
would also like to thank Ulrich von Hörsten (Duisburg-Essen) for his outstanding technical assistance. W. Keune is grateful to S. D. Bader (Argonne) and J. Kirschner (MPI Halle, Germany) for enlightening discussions. F. Scheibel and D. Spoddig are thankful to the AG Farle for the supply of devices. M. Wolloch, P. Mohn, J. Redinger, and D. Suess acknowledge the support by the Austrian Science Fund (FWF) [SFB ViCoM F4109-N28 and F4112-N28]. P. Entel M. E. Gruner, O. Gutfleisch, F. Scheibel, D. Spoddig, and H. Wende acknowledge the support by the Deutsche Forschungsgemeinschaft (DFG) within the priority program SPP 1599 (GR3498/3-2, GU514/6-2, WE2623/12-2, AC63/4-2). Beatriz Roldan acknowledges the support of the US National Science Foundation (NSF-DMR 1207065). This work was supported by the DFG SPP 1681
(WE2623/7-1), FOR 1509 (WE2623/13-2), and by Stiftung Mercator (MERCUR). The authors also appreciate the ample support of computer resources by the Vienna Scientific Cluster (VSC) and the use of the Cray XT6/m and MagnitUDE (DFG grant INST 20876/209-1 FUGG) supercomputers of the Center for Computational Sciences and Simulation (CCSS) at University of Duisburg-Essen. This research used resources of the Advanced Photon Source, a U.S. Department of Energy (DOE) Office of Science User Facility operated for the DOE Office of Science by Argonne National Laboratory under Contract No. DE-AC02-06CH11357. Figures 1, 4, and 5 in this paper were created with help of the VESTA code [126]. The FINDSYM utility of the ISOTROPY software suite [127] was used for structure identification.
[1] M. Fallot, Ann. Phys. (Paris) 10, 291 (1938).

[2] M. Fallot and R. Hocart, Rev. Sci. (Paris) 77, 498 (1939).

[3] G. Shirane, C. W. Chen, P. A. Flinn, and R. Nathans, Phys. Rev. 131, 183 (1963).

[4] G. Shirane, R. Nathans, and C. W. Chen, Phys. Rev. 134, A1547 (1964).

[5] D. Weller, G. Parker, O. Mosendz, E. Champion, B. Stipe, X. Wang, T. Klemmer, G. Ju, and A. Ajan, IEEE Trans. Magn. 50, 1 (2014).

[6] T. Jungwirth, X. Marti, P. Wadley, and J. Wunderlich, Nat. Nano 11, 231 (2016).

[7] B. Yu, M. Liu, P. W. Egolf, and A. Kitanovski, Int. J. Refrig. 33, 1029 (2010).

[8] L. H. Lewis, C. H. Marrows, and S. Langridge, J. Phys. D 49, 323002 (2016)

[9] B. C. Stipe, T. C. Strand, C. C. Poon, H. Balamane, J. A. Katine, J.-L. Li, V. Rawat, H. Nemoto, A. Hirotsune, O. Hellwig, R. Ruiz, E. Dobisz, D. S. Kercher, N. Robertson, T. R. Albrecht, and B. D. Terris, Nat. Photon. 4, 484 (2010).

[10] W. Challener, C. Peng, A. Itagi, D. Karns, W. Peng, Y. Peng, X. Yang, X. Zhu, N. Gokemeijer, Y.-T. Hsia, et al., Nat. Photon. 3, 220 (2009).

[11] H. J. Richter, A. Lyberatos, U. Nowak, R. F. L. Evans, and R. W. Chantrell, J. Appl. Phys. 111, 033909 (2012).

[12] B. V. Budaev and D. B. Bogy, J. Appl. Phys. 112, 034512 (2012).

[13] J.-U. Thiele, S. Maat, and E. E. Fullerton, Appl. Phys. Lett. 82, 2859 (2003).

[14] D. Suess, C. Vogler, C. Abert, F. Bruckner, R. Windl, L. Breth, and J. Fidler, J. Appl. Phys. 117, 163913 (2015).

[15] C. Chappert, A. Fert, and F. N. Van Dau, Nat. Mater. 6, 813 (2007).

[16] R. O. Cherifi, V. Ivanovskaya, L. C. Phillips, A. Zobelli, I. C. Infante, E. Jacquet, V. Garcia, S. Fusil, P. R. Briddon, N. Guiblin, A. Mougin, A. A. Ünal, F. Kronast, S. Valencia, B. Dkhil, A. Barthélémy, and M. Bibes, Nat. Mater. 13, 345 (2014).

[17] Y. Lee, Z. Q. Liu, J. T. Heron, J. D. Clarkson, J. Hong, C. Ko, M. D. Biegalski, U. Aschauer, S. L. Hsu, M. E. Nowakowski, J. Wu, H. M. Christen, S. Salahuddin, J. B. Bokor, N. A. Spaldin, D. G. Schlom, and R. Ramesh, Nat. Commun. 6, 5959 (2015).
[18] L. C. Phillips, R. O. Cherifi, V. Ivanovskaya, A. Zobelli, I C. Infante, E. Jacquet, N. Guiblin, A. A. Ünal, F. Kronast, B. Dkhil, A. Barthélémy, M. Bibes, and S. Valencia, Sci. Rep. 5, 10026 (2015).

[19] Z. Q. Liu, L. Li, Z. Gai, J. D. Clarkson, S. L. Hsu, A. T. Wong, L. S. Fan, M.-W. Lin, C. M. Rouleau, T. Z. Ward, H. N. Lee, A. S. Sefat, H. M. Christen, and R. Ramesh, Phys. Rev. Lett. 116, 097203 (2016).

[20] E. V. Gomonay and V. M. Loktev, Low Temp. Phys. 40, 17 (2014).

[21] X. Marti, I. Fina, C. Frontera, J. Liu, P. Wadley, Q. He, R. J. Paull, J. D. Clarkson, J. Kudrnovský, I. Turek, J. Kuneš, D. Yi, J.-H. Chu, C. T. Nelson, L. You, E. Arenholz, S. Salahuddin, J. Fontcuberta, T. Jungwirth, and R. Ramesh, Nat. Mater. 13, 367 (2014).

[22] N. Matsuzaki, T. Moriyama, M. Nagata, K.-J. Kim, I. Suzuki, T. Taniyama, and T. Ono, Jpn. J. Appl. Phys. 54, 073002 (2015).

[23] T. Moriyama, N. Matsuzaki, K.-J. Kim, I. Suzuki, T. Taniyama, and T. Ono, Appl. Phys. Lett. 107, 122403 (2015).

[24] C. Bordel, J. Juraszek, D. W. Cooke, C. Baldasseroni, S. Mankovsky, J. Minár, H. Ebert, S. Moyerman, E. E. Fullerton, and F. Hellman, Phys. Rev. Lett. 109, 117201 (2012).

[25] T. Usami, I. Suzuki, M. Itoh, and T. Taniyama, Appl. Phys. Lett. 108, 232404 (2016).

[26] M. Annaorazov, K. Asatryan, G. Myalikgulyev, S. Nikitin, A. Tishin, and A. Tyurin, Cryogenics 32, 867 (1992).

[27] J. K. A. Gschneidner and V. K. Pecharsky, Annu. Rev. Mater. Sci. 30, 387 (2000).

[28] M. Manekar and S. B. Roy, J. Phys. D 41, 192004 (2008).

[29] J. Liu, T. Gottschall, K. P. Skokov, J. D. Moore, and O. Gutfleisch, Nat. Mater. 11, 620 (2012).

[30] A. Chirkova, K. Skokov, L. Schultz, N. Baranov, O. Gutfleisch, and T. Woodcock, Acta Mater. 106, 15 (2016).

[31] V. I. Zverev, A. M. Saletsky, R. R. Gimaev, A. M. Tishin, T. Miyanaga, and J. B. Staunton, Appl. Phys. Lett. 108, 192405 (2016).

[32] G. Nikitin, S. A. amd Myalikgulev, M. P. Annaorazon, A. Tyurin, R. W. Myndyev, and S. A. Akopyan, Phys. Lett. A 171, 234 (1992).

[33] E. Stern-Taulats, A. Planes, P. Lloveras, M. Barrio, J.-L. Tamarit, S. Pramanick, S. Majumdar, C. Frontera, and L. Mañosa, Phys. Rev. B 89, 214105 (2014). 
[34] E. Stern-Taulats, A. Gracia-Condal, A. Planes, P. Lloveras, M. Barrio, J. Tamarit, S. Pramanick, S. Majumdar, and L. Manosa, Appl. Phys. Lett. 107, 152409 (2015).

[35] J. B. Staunton, R. Banerjee, M. dos Santos Dias, A. Deak, and L. Szunyogh, Phys. Rev. B 89, 054427 (2014).

[36] Y. Liu, L. C. Phillips, R. Mattana, M. Bibes, A. Barthélémy, and B. Dkhil, Nat. Commun. 7, 11614 (2016).

[37] C. Kittel, Phys. Rev. 120, 335 (1960).

[38] J. S. Kouvel, J. Appl. Phys. 37, 1257 (1966).

[39] J. M. Lommel, J. Appl. Phys. 40, 3880 (1969).

[40] J. B. McKinnon, D. Melville, and E. W. Lee, J. Phys. C 3, S46 (1970).

[41] M. P. Annaorazov, S. A. Nikitin, A. L. Tyurin, K. A. Asatryan, and A. K. Dovletov, J. Appl. Phys. 79, 1689 (1996).

[42] P. Tu, A. J. Heeger, J. S. Kouvel, and J. B. Comly, J. Appl. Phys. 40, 1368 (1969).

[43] J. Ivarsson, G. Pickett, and J. Tóth, Phys. Lett. A 35, 167 (1971).

[44] B. Fogarassy, T. Kemény, L. Pál, and J. Tóth, Phys. Rev. Lett. 29, 288 (1972).

[45] M. E. Gruner, E. Hoffmann, and P. Entel, Phys. Rev. B 67, 064415 (2003)

[46] D. W. Cooke, F. Hellman, C. Baldasseroni, C. Bordel, S. Moyerman, and E. E. Fullerton, Phys. Rev. Lett. 109, 255901 (2012).

[47] G. Ju, J. Hohlfeld, B. Bergman, R. J. M. van de Veerdonk, O. N. Mryasov, J.-Y. Kim, X. Wu, D. Weller, and B. Koopmans, Phys. Rev. Lett. 93, 197403 (2004).

[48] O. N. Mryasov, Phase Transitions 78, 197 (2005).

[49] P. M. Derlet, Phys. Rev. B 85, 174431 (2012).

[50] J. Barker and R. W. Chantrell, Phys. Rev. B 92, 094402 (2015).

[51] L. M. Sandratskii and P. Mavropoulos, Phys. Rev. B 83, 174408 (2011).

[52] J. Kudrnovský, V. Drchal, and I. Turek, Phys. Rev. B 91, 014435 (2015).

[53] A. Deák, E. Simon, L. Balogh, L. Szunyogh, M. dos Santos Dias, and J. B. Staunton, Phys. Rev. B 89, 224401 (2014).

[54] S. Polesya, S. Mankovsky, D. Ködderitzsch, J. Minár, and H. Ebert, Phys. Rev. B 93, 024423 (2016).

[55] R. Y. Gu and V. P. Antropov, Phys. Rev. B 72, 012403 (2005).

[56] M. Richardson, D. Melville, and J. Ricodeau, Phys. Lett. A 46, 153 (1973).

[57] B. K. Ponomarev, Sov. Phys. JETP 36, 105 (1973).

[58] A. Castets, D. Tochetti, and B. Hennion, Physica B+C 86-88, 353 (1977).

[59] Y. Wakisaka, Y. Uemura, T. Yokoyama, H. Asakura, H. Morimoto, M. Tabuchi, D. Ohshima, T. Kato, and S. Iwata, Phys. Rev. B 92, 184408 (2015).

[60] U. Aschauer, R. Braddell, S. A. Brechbühl, P. M. Derlet, and N. A. Spaldin, Phys. Rev. B 94, 014109 (2016).

[61] G. Kresse and J. Hafner, Phys. Rev. B 47, 558 (1993).

[62] G. Kresse and J. Hafner, Phys. Rev. B 49, 14251 (1994).

[63] G. Kresse and J. Furthmüller, Comput. Mater. Sci. 6, 15 (1996).

[64] G. Kresse and J. Furthmüller, Phys. Rev. B 54, 11169 (1996).

[65] P. E. Blöchl, Phys. Rev. B 50, 17953 (1994).

[66] G. Kresse and D. Joubert, Phys. Rev. B 59, 1758 (1999).

[67] J. P. Perdew, K. Burke, and M. Ernzerhof, Phys. Rev. Lett. 77, 3865 (1996).

[68] B. Hammer, L. B. Hansen, and J. K. Nørskov, Phys. Rev. B 59, 7413 (1999).
[69] G. I. Csonka, J. P. Perdew, A. Ruzsinszky, P. H. T. Philipsen, S. Lebègue, J. Paier, O. A. Vydrov, and J. G. Ángyán, Phys. Rev. B 79, 155107 (2009).

[70] J. P. Perdew, K. Burke, and Y. Wang, Phys. Rev. B 54, 16533 (1996).

[71] J. Klimeš, D. R. Bowler, and A. Michaelides, Phys. Rev. B 83, 195131 (2011).

[72] J. P. Perdew and A. Zunger, Phys. Rev. B 23, 5048 (1981).

[73] M. Methfessel and A. T. Paxton, Phys. Rev. B 40, 3616 (1989).

[74] P. E. Blöchl, O. Jepsen, and O. K. Andersen, Phys. Rev. B 49, 16223 (1994).

[75] A. Togo, F. Oba, and I. Tanaka, Phys. Rev. B 78, 134106 (2008).

[76] D. Alfè, Comput. Phys. Commun. 180, 2622 (2009).

[77] See Supplemental Material at http://link.aps.org/supplemental/ 10.1103/PhysRevB.94.174435 for detailed crystallographic information; relations between the electronic structure and the imaginary phonon; experimental details and NRIXS raw data; and further information on thermodynamic properties including a discussion of functional dependency and a BlumeCapel model for the magnetic contributions to $\Delta G$.

[78] S. Salamon et al. (unpublished)

[79] M. Seto, Y. Yoda, S. Kikuta, X. W. Zhang, and M. Ando, Phys. Rev. Lett. 74, 3828 (1995).

[80] W. Sturhahn, T. S. Toellner, E. E. Alp, X. W. Zhang, M. Ando, Y. Yoda, S. Kikuta, M. Seto, C. W. Kimball, and B. Dabrowski, Phys. Rev. Lett. 74, 3832 (1995).

[81] A. I. Chumakov, R. Rüffer, H. Grünsteudel, H. F. Grünsteudel, G. Grübel, J. Metge, O. Leupold, and H. A. Goodwin, Europhys. Lett. 30, 427 (1995).

[82] A. I. Chumakov and W. Sturhahn, Hyperfine Interact. 123/124, 781 (1999).

[83] W. Sturhahn, Hyperfine Interact. 125, 149 (2000).

[84] T. Toellner, Hyperfine Interact. 125, 3 (2000).

[85] S. A. Makhlouf, T. Nakamura, and M. Shiga, J. Magn. Magn. Mater. 135, 257 (1994).

[86] M. R. Ibarra and P. A. Algarabel, Phys. Rev. B 50, 4196 (1994).

[87] S. Jekal, S. H. Rhim, S. C. Hong, W.-j. Son, and A. B. Shick, Phys. Rev. B 92, 064410 (2015).

[88] K. Uebayashi, H. Shimizu, and H. Yamada, Mater. Trans. 47, 456 (2006).

[89] H. Miyajima and S. Yuasa, J. Magn. Magn. Mater. 104-107, 2025 (1992)

[90] S. Yuasa, H. Miyajima, and Y. Otani, J. Phys. Soc. Jpn. 63, 3129 (1994).

[91] S. Yuasa, H. Miyajima, Y. Otani, and A. Sakuma, J. Phys. Soc. Jpn. 64, 4906 (1995).

[92] K. Uebayashi and H. Yamada, J. Magn. Magn. Mater. 310, 1051 (2007).

[93] T. Fukuda and T. Kakeshita, J. Alloys Compd. 563, 192 (2013).

[94] J. Kim, R. Ramesh, and N. Kioussis, Phys. Rev. B 94, 180407 (2016).

[95] R. Witte, R. Kruk, M. E. Gruner, R. A. Brand, D. Wang, S. Schlabach, A. Beck, V. Provenzano, R. Pentcheva, H. Wende, and H. Hahn, Phys. Rev. B 93, 104416 (2016).

[96] S. Yuasa, H. Miyajima, Y. Otani, G. Masada, N. Wakabayashi, and K. Tajima, J. Phys. Soc. Jpn. 64, 3153 (1995).

[97] R. C. Wayne, Phys. Rev. 170, 523 (1968).

[98] L. I. Vinokurova, A. V. Vlasov, and M. Pardavi-Horváth, Phys. Status Solidi B 78, 353 (1976). 
[99] V. Kuncser, R. Nicula, U. Ponkratz, A. Jianu, M. Stir, E. Burkel, and G. Filoti, J. Alloys Compd. 386, 8 (2005).

[100] T. Asada and K. Terakura, Phys. Rev. B 46, 13599 (1992).

[101] R. Hafner, D. Spišák, R. Lorenz, and J. Hafner, Phys. Rev. B 65, 184432 (2002).

[102] A. V. Ruban and I. A. Abrikosov, Rep. Prog. Phys. 71, 046501 (2008).

[103] I. I. Mazin, M. D. Johannes, L. Boeri, K. Koepernik, and D. J. Singh, Phys. Rev. B 78, 085104 (2008).

[104] J. J. Pulikkotil, S. Auluck, P. K. Rout, and R. C. Budhani, J. Phys.: Condens. Matter 24, 096003 (2012).

[105] J. M. Lommel and J. S. Kouvel, J. Appl. Phys. 38, 1263 (1967).

[106] L. Vinokurova, E. Kulatov, A. Vlasov, and M. Pardavi-Horvath, J. Phys. Colloques 49, C8-99 (1988).

[107] A. Szajek and J. Morkowski, Physica B: Condens. Matter 193, 81 (1994)

[108] M. A. Uijttewaal, T. Hickel, J. Neugebauer, M. E. Gruner, and P. Entel, Phys. Rev. Lett. 102, 035702 (2009).

[109] R. Niemann, U. K. Rößler, M. E. Gruner, O. Heczko, L. Schultz, and S. Fähler, Adv. Eng. Mater. 14, 562 (2012).

[110] M. E. Gruner, S. Fähler, and P. Entel, Phys. Status Solidi B 251, 2067 (2014).

[111] R. Barua, F. Jiménez-Villacorta, and L. H. Lewis, Appl. Phys. Lett. 103, 102407 (2013).

[112] M. E. Gruner, W. Keune, B. Roldan Cuenya, C. Weis, J. Landers, S. I. Makarov, D. Klar, M. Y. Hu, E. E. Alp, J. Zhao, M. Krautz, O. Gutfleisch, and H. Wende, Phys. Rev. Lett. 114, 057202 (2015).
[113] B. Fultz, Prog. Mater. Sci. 55, 247 (2010).

[114] T. Hickel, B. Grabowski, F. Körmann, and J. Neugebauer, J. Phys.: Condens. Matter 24, 053202 (2012).

[115] B.-T. Wang, P. Zhang, R. Lizárraga, I. Di Marco, and O. Eriksson, Phys. Rev. B 88, 104107 (2013).

[116] A. I. Zakharov, A. M. Kadomtseva, R. Z. Levitin, and E. G. Ponyatovskii, Sov. Phys. JETP 19, 1348 (1964).

[117] R. Z. Levitin and B. K. Ponomarev, Sov. Phys. JETP 23, 984 (1966).

[118] A. Szajek and J. Morkowski, J. Magn. Magn. Mater. 115, 171 (1992).

[119] J. A. Ricodeau and D. Melville, J. Phys. F 2, 337 (1972).

[120] A. X. Gray, D. W. Cooke, P. Krüger, C. Bordel, A. M. Kaiser, S. Moyerman, E. E. Fullerton, S. Ueda, Y. Yamashita, A. Gloskovskii, C. M. Schneider, W. Drube, K. Kobayashi, F. Hellman, and C. S. Fadley, Phys. Rev. Lett. 108, 257208 (2012).

[121] D. C. Langreth and M. J. Mehl, Phys. Rev. Lett. 47, 446 (1981).

[122] H. Takahashi, M. Araidai, S. Okada, and K. Shiraishi, J. Magn. Soc. Jpn. 40, 77 (2016).

[123] K. Yosida, Theory of Magnetism, Springer Series in Solid-State Sciences Vol. 122 (Springer, Berlin, Heidelberg, New York, 1996).

[124] P. W. Anderson, Phys. Rev. 86, 694 (1952).

[125] R. Kubo, Phys. Rev. 87, 568 (1952).

[126] K. Momma and F. Izumi, J. Appl. Crystallogr. 44, 1272 (2011).

[127] H. T. Stokes and D. M. Hatch, J. Appl. Crystallogr. 38, 237 (2005). 